\title{
A WIDE-FIELD NEAR- AND MID-INFRARED CENSUS OF YOUNG STARS IN NGC 6334
}

\author{
S. Willis ${ }^{1}$, M. Marengo ${ }^{1}$, L. Allen ${ }^{2}$, G. G. Fazio ${ }^{3}$, H. A. Smith ${ }^{3}$, and S. Carey ${ }^{4}$ \\ ${ }^{1}$ Department of Physics and Astronomy, Iowa State University, Ames, IA 50010, USA; swillis@ cfa.harvard.edu \\ 2 National Optical Astronomy Observatories, Tucson, AZ 85719, USA \\ ${ }^{3}$ Harvard-Smithsonian Center for Astrophysics, Cambridge, MA 02138, USA \\ ${ }^{4}$ Spitzer Science Center, California Institute of Technology, Pasadena, CA 91125, USA \\ Received 2013 June 28; accepted 2013 September 25; published 2013 November 8
}

\begin{abstract}
This paper presents a study of the rate and efficiency of star formation in the NGC 6334 star-forming region. We obtained observations at $J, H$, and $K_{s}$ taken with the NOAO Extremely Wide-Field Infrared Imager and combined them with observations taken with the Infrared Array Camera (IRAC) on the Spitzer Space Telescope at wavelengths $=3.6,4.5,5.8$, and $8.0 \mu \mathrm{m}$. We also analyzed previous observations taken at $24 \mu \mathrm{m}$ using the Spitzer MIPS camera as part of the MIPSGAL survey. We have produced a point source catalog with $>700,000$ entries. We have identified 2283 young stellar object (YSO) candidates, 375 Class I YSOs, and 1908 Class II YSOs using a combination of existing IRAC-based color classification schemes that we have extended and validated to the nearIR for use with warm Spitzer data. We have identified multiple new sites of ongoing star formation activity along filamentary structures extending tens of parsecs beyond the central molecular ridge of NGC 6334. By mapping the extinction, we derived an estimate for the gas mass, $2.2 \times 10^{5} M_{\odot}$. The heavy concentration of protostars along the dense filamentary structures indicates that NGC 6334 may be undergoing a "mini-starburst" event with $\Sigma_{\mathrm{SFR}}>8.2 M_{\odot} \mathrm{Myr}^{-1} \mathrm{pc}^{-2}$ and SFE $>0.10$. We have used these estimates to place NGC 6334 in the Kennicutt-Schmidt diagram to help bridge the gap between observations of local low-mass star-forming regions and star formation in other galaxies.
\end{abstract}

Key words: ISM: individual objects (NGC 6334) - stars: formation

Online-only material: color figures, machine-readable tables

\section{INTRODUCTION}

Active star formation in other galaxies can be inferred from several indicators of the presence of short-lived massive stars, including strong UV flux and $\mathrm{H} \alpha$ emission from excited hydrogen gas. Although these massive stars dominate the observable emission, they represent only a tiny fraction of the total stellar mass being formed; the majority of this is contained in much more numerous low-mass stars. Empirical relations, such as the Kennicutt-Schmidt law (Kennicutt 1998), have matched the observed surface density of total rate of star formation to the surface density of gas in other galaxies. Recent detailed studies of nearby Galactic star-forming regions obtained a complete census of the young stellar content and found star formation rates orders of magnitude higher than predicted by these empirical relations (Evans et al. 2009). One possible explanation for this discrepancy is that the dense gas most relevant to the star formation process appears diluted in extragalactic observations due to the more abundant diffuse clouds. In contrast, the spatial resolution in Galactic starforming regions is sufficient for the dense gas to fill the beam (Heiderman et al. 2010).

Another important difference between the nearby, wellstudied star-forming regions and star-forming regions observed in other galaxies is that the nearby regions are primarily forming low-mass stars. Due to the feedback between massive stars and their environments, it is important to also study massive Galactic regions analogous to the star-forming regions seen in other galaxies that are near enough to obtain an accurate census of star formation activity from high-mass stars to below $1 M_{\odot}$.

One such Galactic region is NGC 6334, or the Cat's Paw Nebula. The current understanding of NGC 6334 is well summarized in a recent review by Persi \& Tapia (2008). NGC 6334 is a large $\left(M>10^{5} M_{\odot}\right)$ molecular cloud complex located at $l=351^{\circ}, b=0.7$. OB stars have been detected both in clusters and isolated throughout the entire molecular cloud complex (Neckel 1978), with a recent $U B V R$ survey turning up 150 OB stars (Russeil et al. 2012). The central portion of the cloud consists of a dense dusty ridge extending approximately $10 \mathrm{pc}$ parallel to the Galactic plane hosting multiple sites of massive star formation. Historically different nomenclature has been used to describe the brightest radio and far-infrared (FIR) sources in this region. We are using the $\mathrm{A}, \mathrm{C}, \mathrm{D}, \mathrm{E}$, and $\mathrm{F}$ radio source labels from Rodriguez et al. (1982), the roman numeral I, II, III, IV, and V FIR source names from McBreen et al. (1979), and the $\mathrm{I}(\mathrm{N})$ reference from Gezari (1982). We will refer to sources that are detected in both the radio and FIR continuum by both designations, e.g., NGC 6334 I/F. The main features of NGC 6334 are shown in Figure 1. NGC $6334 \mathrm{I}(\mathrm{N})$ and I/F are among the farthest north in the ridge and are also extremely young. I(N), a deeply embedded protocluster of massive stars, has recently been studied extensively with observatories such as Herschel and the Sub-Millimeter Array (Brogan et al. 2009). Just to the south is NGC $6334 \mathrm{II} / \mathrm{D}$, a small well-defined H II region. NGC $6334 \mathrm{~V}$ is another young FIR source that is driving a massive molecular outflow (Fischer et al. 1982). NGC 6334 III/C and NGC 6334 IV/A are also joint FIR and radio sources, each surrounding a late O-type star and having $L_{\text {bol }}>10^{5} L_{\odot}$.

This diversity of environments within NGC 6334 provides a unique laboratory to study massive star formation at all stages of pre-main-sequence evolution. In addition, as NGC 6334 is one of the closest sites of massive star formation to the Sun (1.61 $\pm 0.08 \mathrm{kpc}$; Persi \& Tapia 2008), we are also able to characterize the large population of low-mass stars the cloud 


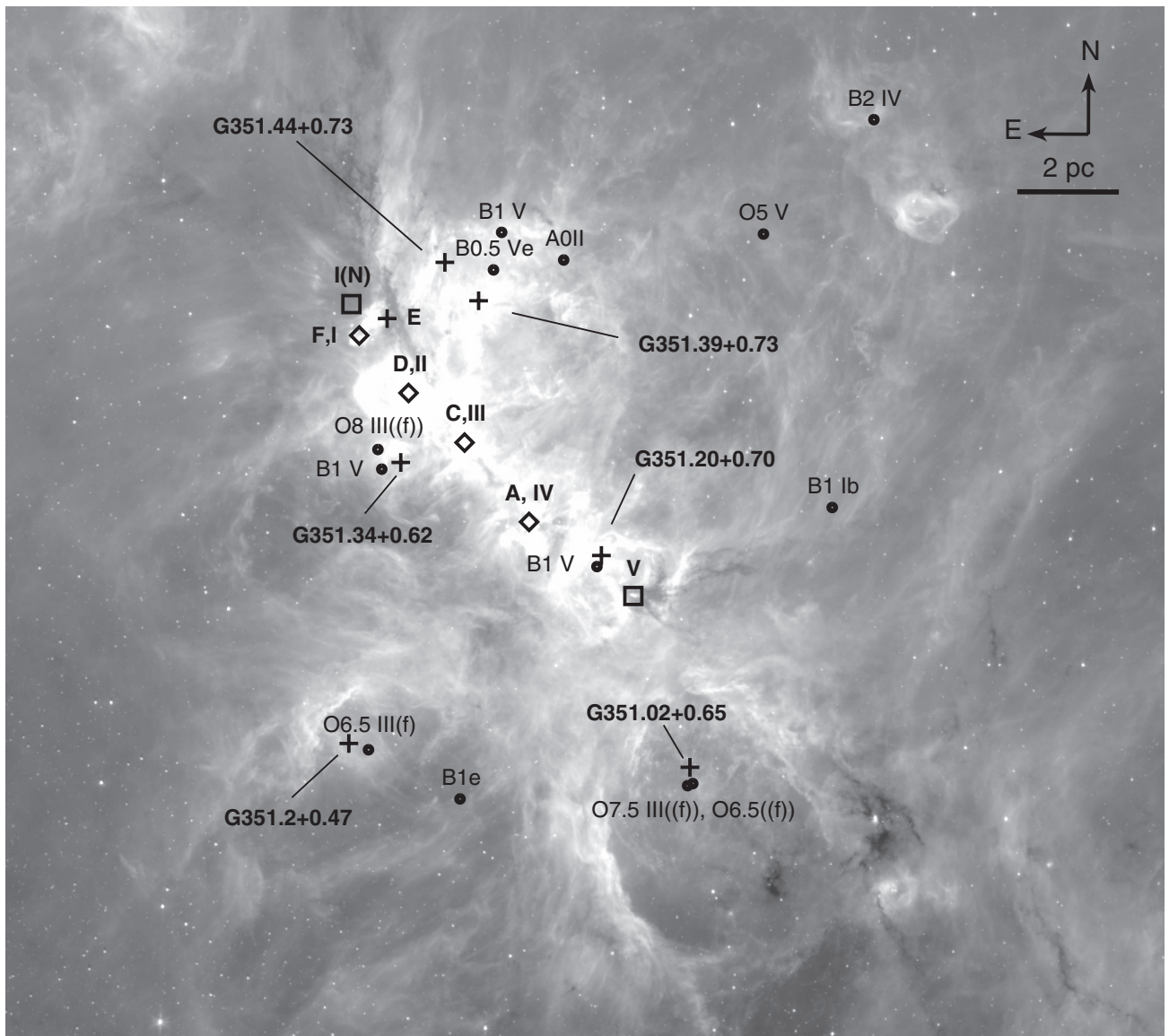

Figure 1. Spitzer IRAC $8.0 \mu \mathrm{m}$ image of NGC 6334 with the FIR sources from McBreen et al. (1979) and Gezari (1982) (I, I(N), II, III, IV, V) and the radio sources (A, C, D, E, F) from Rodriguez et al. (1982) marked. Sources identified only in the radio are marked with plusses, sources identified only in the FIR are marked with boxes, and sources that are detected in both the radio and FIR are marked with diamonds. The massive stars believed responsible for the excitation of the optical nebulae are also marked with dots and labeled by spectral type as given in Persi \& Tapia (2008 and references therein). The image is centered at $17^{\mathrm{h}} 20^{\mathrm{m}} 01^{\mathrm{s}}-35^{\mathrm{d}} 53^{\mathrm{m}} 11^{\mathrm{s}}$. The scale bar is approximately 4.2 in length, or $2 \mathrm{pc}$ at the assumed distance of $1.6 \mathrm{kpc}$.

is producing. Prior to this work, there have been no deep, extensive near-infrared (near-IR) and mid-infrared (mid-IR) surveys covering the entire NGC 6334 star-forming complex in a systematic way. Shallow observations in the mid-IR include the Galactic Legacy Infrared Mid-Plane Survey Extraordinaire (GLIMPSE; Benjamin et al. 2003; Churchwell et al. 2009) and the MIPS Inner Galactic Plane Survey (MIPSGAL; Carey et al. 2009) programs using the Infrared Array Camera (IRAC; Fazio et al. 2004) and the Multiband Imaging Photometer for Spitzer (MIPS; Rieke et al. 2004) on board the Spitzer Space Telescope (Werner et al. 2004). Deep, ground-based near-IR observations have targeted small portions of the cloud (e.g., Tapia et al. 1996), and shallow low-resolution studies of the whole cloud have also been completed (e.g., Straw \& Hyland 1989). In this paper, we present the analysis of new deep observations with Spitzer IRAC as well as a large deep survey of the entire star-forming region in the near-IR with the National Optical Astronomy Observatory (NOAO) Extremely Wide-Field Infrared Imager (NEWFIRM) camera (Probst et al. 2004) on the Blanco $4 \mathrm{~m}$ telescope at Cerro Tololo Inter-American Observatory (CTIO). We also incorporate our analysis of the publicly available $24 \mu \mathrm{m}$ data from the MIPSGAL survey program.

In this comprehensive study, we have obtained a census of the young stellar population of this massive and complex region extending from the high-mass protostars to below $1 M_{\odot}$. We identify the sources based on the morphology of their spectral energy distribution (SED). Sources that have a rising spectrum toward long wavelengths are identified as Class I young stellar objects (YSOs) and sources that have a flatter, or negative slope toward longer wavelengths are identified as Class II YSOs.

Combined with our precise mapping of extinction in the region, we have extended the empirical relations between gas surface density and star formation rate to a broader range of masses. This is the first paper of a series in which we will examine star formation efficiency in five other massive Galactic star-forming regions that we have also observed with Spitzer and NEWFIRM.

In Section 2 of this paper, we describe our observations, and in Section 3 we present our methodology for the data reduction and photometry. In Section 4, we present our method to identify the candidate YSO population, and in Section 5 we delve into the derived properties of the star formation activity in NGC 6334.

\section{OBSERVATIONS}

Figure 2 shows the footprint of our observations of NGC 6334. The cloud is observed in its entirety with our near-IR (NEWFIRM) and mid-IR (IRAC) data. Archival observations from the MIPSGAL survey cover all but the northwest portion of the cloud. Approximately $0.8 \mathrm{deg}^{2}$ were observed from $J$ band to $8.0 \mu \mathrm{m}$.

\subsection{Spitzer Observations}

We observed a 1.2 by 0.9 region covering NGC 6334 (shown in Figure 3) using IRAC during Spitzer's cryogenic mission on 


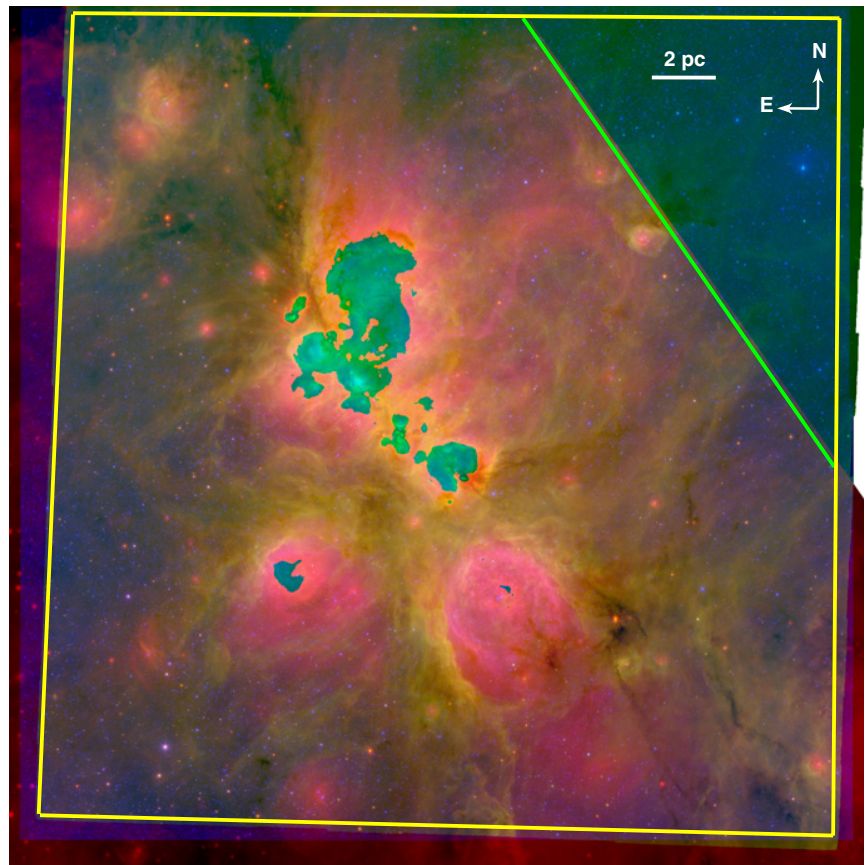

Figure 2. Three-color image of NGC 6334 with $J$ band for blue, $8.0 \mu \mathrm{m}$ for green, and $24 \mu \mathrm{m}$ red. The cyan colored regions near the center mark where the $24 \mu \mathrm{m}$ mosaics are saturated. The region outlined in yellow denotes the area with full seven band coverage, $J, H$, and $K_{s}$ band from NEWFIRM, and $3.6,4.5,5.8$, and $8.0 \mu \mathrm{m}$ with IRAC. The area in the northwest corner above the green line is missing MIPS $24 \mu \mathrm{m}$ coverage. The image is centered at $17^{\mathrm{h}} 20^{\mathrm{m}} 01^{\mathrm{s}}-35^{\mathrm{d}} 53^{\mathrm{m}} 11^{\mathrm{s}}$. The scale bar is approximately 4.2 in length, or $2 \mathrm{pc}$ at the assumed distance of $1.6 \mathrm{kpc}$.

2008 September 21 (PID 30154) at 3.6, 4.5, 5.8, and $8.0 \mu \mathrm{m}$. We mapped the region with a grid of 13 by 11 tiles in two epochs spaced by $6 \mathrm{hr}$ with three dither positions per tile per epoch. The two epochs were intented to be scheduled six months apart (to allow variability studies), but were then executed back to back to ensure completion of the data set before exhaustion of the cryogen. The region observed corresponds to approximately 33 by $25 \mathrm{pc}$ at a distance of $1.6 \mathrm{kpc}$.

We used IRAC's $12 \mathrm{~s}$ frame time high dynamic range (HDR) mode, acquiring consecutive individual observations with exposure times of 0.4 and $10.4 \mathrm{~s}$. The long exposures probe the faint stellar population in the cloud while the addition of the short exposure observations allow for the recovery of bright sources that are saturated in the long exposure frames. Due to the short interval between the two epochs, both epochs were combined to produce the final mosaics. The 858 individual Basically Calibrated Frames (BCD; processed with the Spitzer IRAC pipeline version S18.7.0) for each exposure time were mosaicked together using the IRACproc package (Schuster et al. 2006) to perform outlier rejection and bad pixel masking. IRACproc is a Perl Data Language wrapper script for the standard Spitzer Science Center mosaicking software MOPEX (Makovoz \& Khan 2005), which has been enhanced for better cosmic ray rejection. Each mosaic was re-sampled from the native IRAC resolution of $1^{\prime \prime} .22$ pixel $^{-1}$ to 0.86267 pixel $^{-1}$ and projected onto a common world coordinate system grid. The final mosaics combined a median of six dither positions per pixel for an effective integration time of $62.4 \mathrm{~s} \mathrm{pixel}^{-1}$ in each long exposure mosaic and $2.4 \mathrm{~s}$ in the short exposure mosaic. The bright nebular emission along the central ridge of NGC 6334 severely saturated the central portion of the long exposure mosaic at $8.0 \mu \mathrm{m}$ but remains unsaturated in the short exposure mosaic.

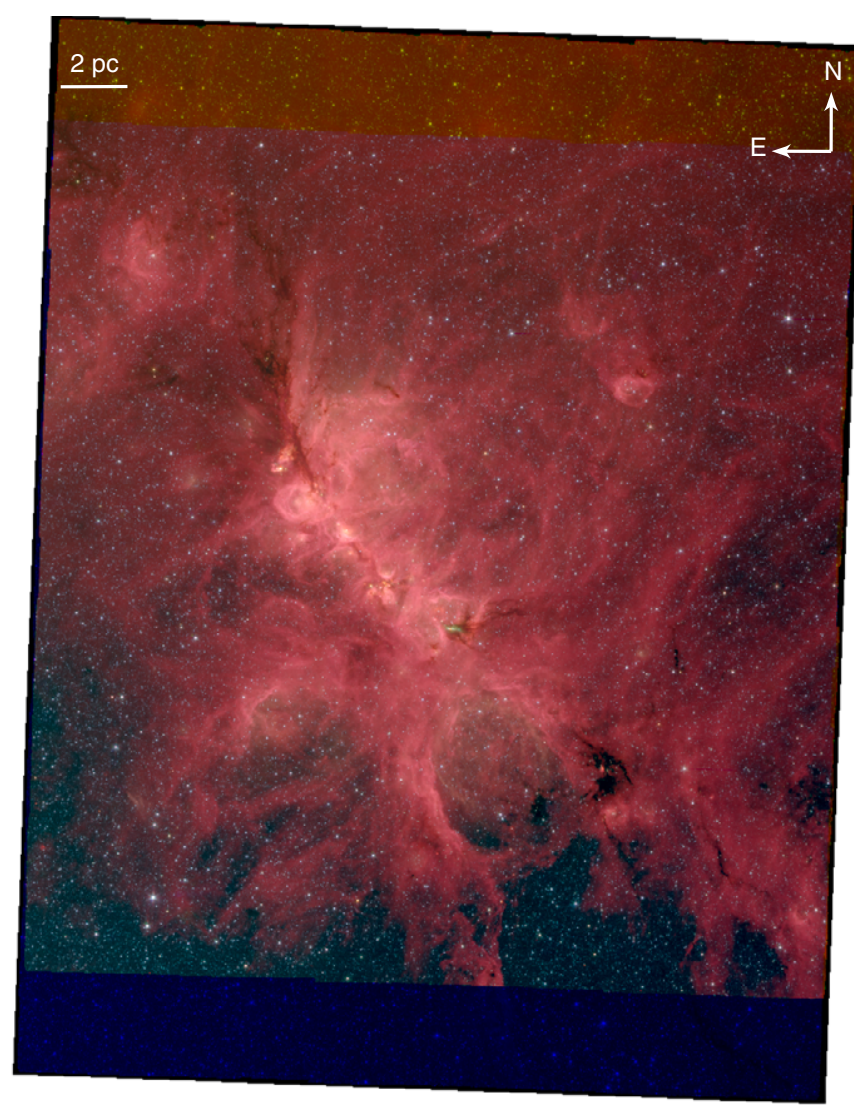

Figure 3. Three-color image of the observed IRAC field using the HDR mosaics in different bands. We have generated the HDR mosaics by smoothly merging the short frame mosaics to fill in the saturated areas in the long frame mosaics. The color coding is blue, green, and red for $\lambda=3.6,4.5$, and $8.0 \mu \mathrm{m}$, respectively. The image is centered at $17^{\mathrm{h}} 20^{\mathrm{m}} 01^{\mathrm{s}}-35^{\mathrm{d}} 53^{\mathrm{m}} 11^{\mathrm{s}}$. The scale bar is approximately 4.2 in length, or $2 \mathrm{pc}$ at the assumed distance of $1.6 \mathrm{kpc}$.

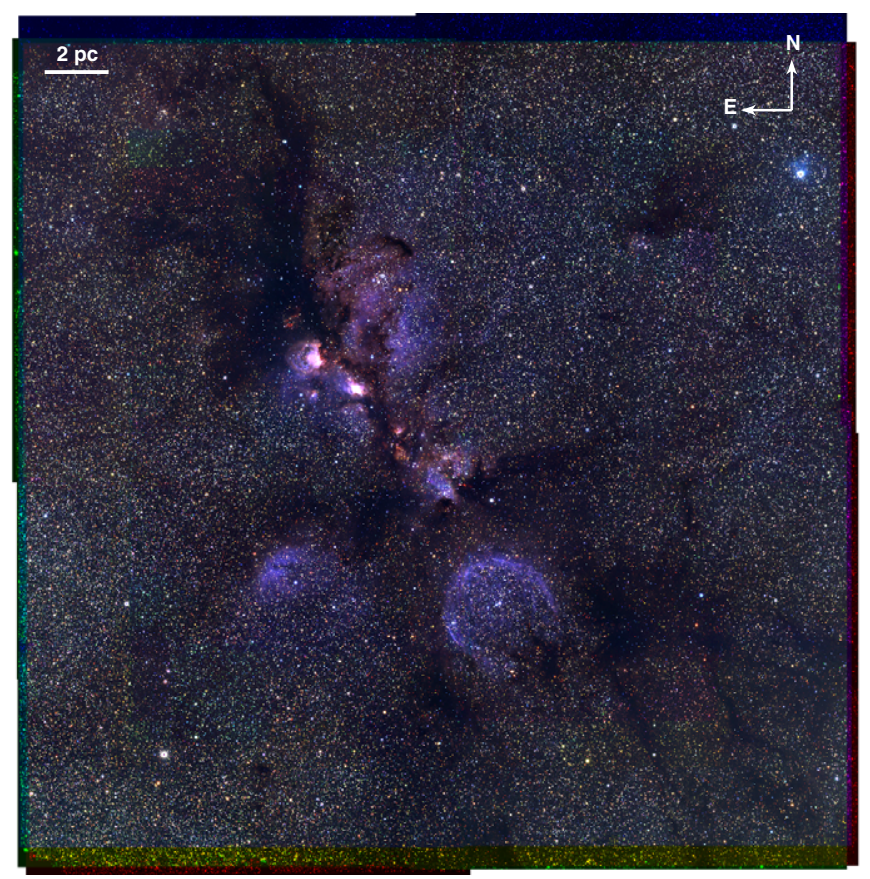

Figure 4. Three-color image of the central pointing NEWFIRM field. The color coding is blue, green, and red for the $J, H$, and $K_{s}$ bands, respectively. The image is centered at $17^{\mathrm{h}} 20^{\mathrm{m}} 01^{\mathrm{s}}-35^{\mathrm{d}} 53^{\mathrm{m}} 11^{\mathrm{s}}$. The scale bar is approximately 4.2 in length, or $2 \mathrm{pc}$ at the assumed distance of $1.6 \mathrm{kpc}$. 
We also retrieved the archival MIPSGAL $24 \mu \mathrm{m}$ observations of NGC 6334 obtained as part of the MIPSGAL survey. The central ridge running from the northeast to the southwest of NGC 6334 is saturated in the MIPSGAL $24 \mu \mathrm{m}$ images. The available observations of NGC 6334 span across two MIPSGAL mosaics, which are shown combined in Figure 2. The upper northwest corner of NGC $6334(b>1.0)$ was not covered by the MIPSGAL survey. Full details of the MIPSGAL observations can be found in Carey et al. (2009). The point-spread function (PSF) of the $24 \mu \mathrm{m}$ mosaics is $6^{\prime \prime}$ FWHM and the median exposure per pixel in these MIPSGAL mosaics was $30 \mathrm{~s}$.

\subsection{NEWFIRM Observations}

Figure 4 shows our near-IR images of NGC 6334 acquired with the NEWFIRM camera on the Blanco $4 \mathrm{~m}$ telescope at CTIO during the 2010A semester from 2010 May 24 to 30. The NEWFIRM camera, designed to quickly map large areas of the sky, contains four InSb $2048 \times 2048$ pixel arrays arranged in a $2 \times 2$ pattern with a $28^{\prime}$ field of view and an approximately $1^{\prime}$ gap between the CCDs. The detector has a pixel scale of 0.4 pixel $^{-1}$.

To match the 1.2 by 0.9 sky coverage of our IRAC mosaics, we used a 2 by 2 central pointing mapping routine to divide NGC 6334 into four quadrants plus an overlapping central region. Our observations were taken with a random dither offset large enough to fill in the $1^{\prime}$ gap between CCDs. For each quadrant we took $60 \mathrm{~s}$ exposures at 11 dither positions. Each dither position was a combination of multiple co-added exposures to achieve longer exposure times while avoiding the non-linear regime of the detector. For the $J$ band we used two co-added $30 \mathrm{~s}$ exposures, for the $H$ band we used three co-added $20 \mathrm{~s}$ exposures, and for the $K_{s}$ band we used six co-added $10 \mathrm{~s}$ exposures. In the central pointing, the $J$-band image used $20 \mathrm{~s}$ exposures and three co-adds, and the $H$ and $K_{s}$ bands used a slightly shallower $4 \mathrm{~s}$ exposures and eight co-adds. The sky background was measured by taking three dithered sky observations $2^{\circ}$ off field.

Standard processing of dark frame subtraction, flat fielding, sky-subtraction, and bad pixel masking was performed by the NEWFIRM Science Pipeline (Dickinson \& Valdes 2009; Swaters et al. 2009) to produce five stacked composite images for each near-IR band. The final stacked images retain the detector pixel scale of 0.4 pixel $^{-1}$. Variable seeing conditions caused the final observed point source FWHM in the stacked images to range from approximately $0.9-1{ }^{\prime \prime} .5$. The $H$-band observations were taken during the best seeing conditions and have the smallest FWHM, and the $K_{s}$ images have the largest FWHM. Even in the most crowded portions of the region observed, however, the difference in effective resolution at the different NEWFIRM bands has a minimal effect on later sourcematching.

\section{SOURCE FINDING AND PHOTOMETRY}

We used DAOPHOT (Stetson 1987) to perform PSF-fitting photometry in each final mosaic individually; the long- and short-exposure mosaics for each of the four IRAC bands, the five pointings per band from NEWFIRM, and the two available MIPSGAL $24 \mu \mathrm{m}$ mosaics.

The PSF for each image was derived from a minimum of 10 bright, unsaturated, and relatively isolated stars that are well separated from the extended nebular emission. The PSF obtained for the short-exposure IRAC frames was also used for the corresponding long-exposure mosaic. The instrumental PSF was defined in a 24 ". $4 \times 24^{\prime \prime} .4$ box $(20 \times 20$ IRAC native pixels $)$ chosen to be approximately 10 times larger than the observed FWHM of an individual point source and therefore include the vast majority of the flux of a point source.

Due to the crowded Galactic field at NGC 6334's latitude $(b=0.7)$, even in the short exposure mosaics most of the bright sources are surrounded by other point sources within the 24 ". 4 box used to define the PSF. If left alone, the flux from these nearby point sources will skew the derived PSF and introduce additional systematic error into the photometry. We utilized a multiple-iteration method to converge on a satisfactory PSF for each frame. We began with a PSF composed purely of an analytical Lorentz function and used it to subtract the neighboring sources from each of the 10 or more selected PSF stars. The source-subtracted image was then re-examined, and the PSF re-derived, allowing corrections to the analytic function based on the observed shape of the PSF. We progressed through four total iterations of fitting and subtracting the neighboring sources and then redefining the PSF from the PSF stars in the neighbor-subtracted frames.

The high variability of the background nebular emission at IRAC wavelengths over small spatial scales proved challenging for a single point source detection threshold to be used across the entire mosaic. To better enable the detection of all faint point sources regardless of position in the mosaic, we used a multiple-iteration background subtraction method similar to the procedure in Strafella et al. (2010). We first used the photometry for the preliminary source list to subtract all point sources from each frame. The source-subtracted image was then median filtered with a $51 \times 51$ pixel box to produce an image of the background emission. This background image was then subtracted from the original mosaic. The background subtracted mosaics were then used to refine the location of the point sources and identify the ones that were lost in the background diffuse emission. For our NEWFIRM images, the variability of the sky background was low enough that we did not need to use the background subtraction method to achieve comparable completeness.

The resulting source list was fed into DAOPHOT's ALLSTAR routine to PSF-fit each source. To reduce the effect of crowding, only the flux within a 3 pixel radius was used to fit the model PSF, corresponding to $2^{\prime \prime} .6$ in the IRAC images and $1^{\prime \prime} .2$ in the NEWFIRM images. Detected sources that deviate from the expected point source shape by more than $5 \%$ were rejected. The majority of rejected sources were spurious detections along the extended emission, although they also can indicate partially resolved background galaxies as well as blended PSFs from pairs of stars with extremely small angular separation. An example of the residuals left after subtracting the derived PSF for a representative crowded field can be seen in Figure 5.

We ran the subtracted mosaics through DAOPHOT for two subsequent passes to find point sources that were missed during the first pass. Occasionally when running a second or third iteration, the DAOPHOT find algorithm would detect bright pixels in the residuals of subtracted sources as a new legitimate point source. In all bands, any source detected in the second or third photometry iteration that had a centroid position matching within less than $2^{\prime \prime}$ (approximately the mean FWHM of the IRAC point sources) of a source from a previous iteration was considered a duplicate detection. The duplicate sources identified in this way were rejected from further analysis. 


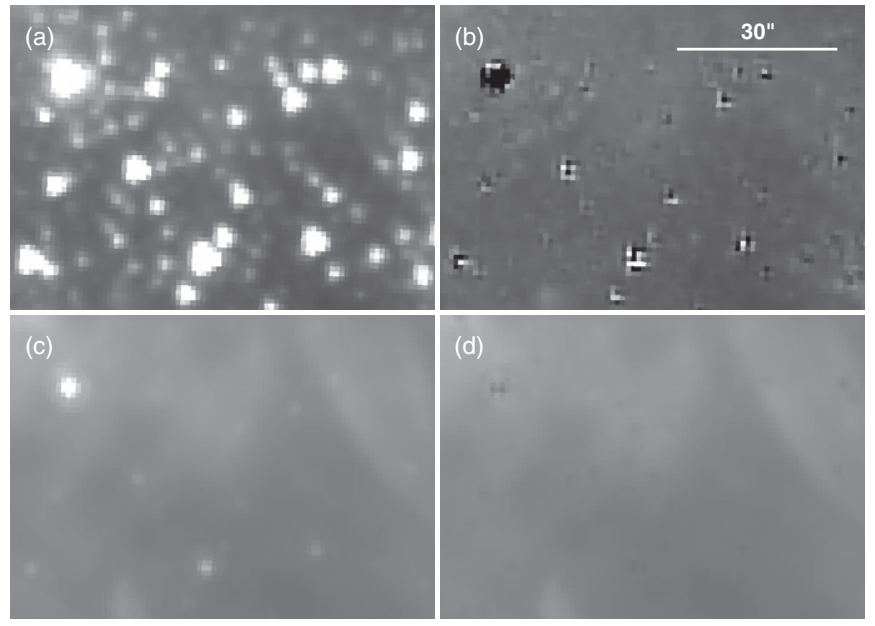

Figure 5. Example of PSF subtraction in a representative crowded field in NGC 6334. The left panels show the long exposure mosaics at $\lambda=3.6 \mu \mathrm{m}$ (a) and $8.0 \mu \mathrm{m} \mathrm{(c).} \mathrm{The} \mathrm{right} \mathrm{panels} \mathrm{show} \mathrm{the} \mathrm{result} \mathrm{of} \mathrm{point} \mathrm{source} \mathrm{subtraction}$ using the PSF derived from the isolated bright stars in each corresponding short exposure mosaic for $\lambda=3.6 \mu \mathrm{m}$ (b) and $8.0 \mu \mathrm{m}$ (d).

Sources brighter than magnitude 9.2 are saturated in the longexposure mosaic at $3.6 \mu \mathrm{m}$. The saturation limit is 9.0, 6.5, and $6.5 \mathrm{mag}$ for $4.5,5.8$, and $8.0 \mu \mathrm{m}$, respectively. Where a source in the long-exposure mosaic frame matched within 2.0 of a source with a short-exposure magnitude brighter than the saturation limit, the short exposure photometry was used.

The same method described above was used to compile a preliminary source list for each of the five images for the three near-IR bands, $J, H$, and $K_{s}$. In each photometric band, the NEWFIRM pointings overlapped by approximately $3^{\prime}$, causing many sources to be detected in more than one NEWFIRM stacked image. Sources with centroid positions matching within 0 ". 8 in different stacked images were combined; we retained the average magnitude weighted by the exposure time contributing to that source in each stacked image. Point sources detected in two or fewer stacked images (out of the maximum possible 11 for each mosaic) were rejected as being likely artifacts left over after pipeline processing. As a result, $120 \mathrm{~s}$ is the minimum exposure time for each NEWFIRM source included in the point source catalog. The median point source exposure time was $600 \mathrm{~s}$ for $J$ and $K_{s}$ bands and $480 \mathrm{~s}$ for $H$ band.

We also applied the same photometry process to the MIPSGAL $24 \mu \mathrm{m}$ mosaics. Similar to the $8.0 \mu \mathrm{m}$ mosaics from IRAC, the find algorithm identified many small bright areas along the extended emission as point sources. Larger restrictions were placed on the DAOPHOT PSF-fitting parameters of detected

$24 \mu \mathrm{m}$ point sources to decrease the number of spurious detections. After these cuts, 991 point sources in the MIPSGAL mosaics have a centroid matching within $1^{\prime \prime}$ of an IRAC/ NEWFIRM source. Due to the issues with saturation, there is a large difference in the completeness of the MIPS portion of the source catalog across the region.

We used the short-exposure mosaic to calibrate the instrumental PSF photometry in each IRAC band against 100 relatively isolated and bright stars measured with aperture photometry in PhotVis (Gutermuth et al. 2004). The same procedure was used to calibrate the $24 \mu \mathrm{m}$ photometry. To determine the zero point for our near-IR photometry, we matched up bright isolated stars from our NEWFIRM catalogs with sources from the
Two Micron All Sky Survey (2MASS; Skrutskie et al. 2006) source list. In the higher source-density portions of the field, the 2MASS photometry suffers more from crowding and blending due to the lower survey resolution (2".4 versus 0 .'9), leading to a larger number of sources with artificially bright magnitudes. To decrease the flux contamination effect from this crowding, sources for the near-IR photometric calibration were carefully chosen from uncrowded portions of the mosaics.

\subsection{NGC 6334 Point Source Catalog}

We created the final source list catalog by spatially matching the detected sources across the different bands. We first matched sources detected with the same instrument, $J, H$, and $K_{s}$ for NEWFIRM and 3.6, 4.5, 5.8, and $8.0 \mu \mathrm{m}$ for IRAC. Our crossband correlation method first calculates whether there is an overall systematic shift in source centroid positions between the input frames, and then looks for sources with shifted centroid positions matching between bands. The NEWFIRM sources were matched if their centroid positions differed by less than 0 '.8. For IRAC, due to the larger pixel scale and broader FWHM, we required sources to have centroids that matched within $1^{\prime \prime} .0$ between bands. To combine the two catalogs together, we used the larger 1 ."0 matching criterion.

To further increase the reliability of our point source cata$\log$, we first required simultaneous detection in two adjacent bands (e.g., $H$ and $K_{s}$ on NEWFIRM, or 3.6 and $4.5 \mu \mathrm{m}$ with IRAC). Following that, we also included sources at the edges of the IRAC mosaics only visible at 3.6 and $5.8 \mu \mathrm{m}$ or at 4.5 and $8.0 \mu \mathrm{m}$ bands. Finally, due to the large number of very red sources in the field, we also retained photometry for approximately $7000 K_{s}$-band sources lacking detection at $J$ or $H$, but that matched within 0.5 with a source detected in two or more adjacent IRAC bands. Histograms of all the sources kept for the NGC 6334 point source catalog are presented in Figure 6. The completeness limit for each band was estimated from the turnover point in each histogram and the limiting magnitude reported is the faintest source detected at the $5 \sigma$ level or higher (a maximum photometric error of 0.217 magnitudes). Sample entries of the NGC 6334 Point Source Catalog can be seen in Table 1. The full version is available in electronic format. A statistical summary of the Point Source Catalog can be found in Table 2.

\section{YSO IDENTIFICATION}

Observational methods of identifying YSOs have been derived from evolutionary models and comparison to observed known YSOs. One early method was to fit the slope $\alpha$ of $\log \left(\lambda F_{\lambda}\right)$ versus $\log (\lambda)$ between 2 and $24 \mu \mathrm{m}$ (Lada 1987). Other methods have referenced color selection criteria for YSO stars (e.g., Gutermuth et al. 2009). We used the full range of observed wavelengths from $J$ band to $24 \mu \mathrm{m}$ for the purpose of identifying YSO candidates in our point source catalog. The selection is based on color and magnitude criteria, similar to those defined in Gutermuth et al. (2009). We have however adapted these selection criteria to take into account the greater distance of NGC 6334 and the higher level of contamination due to crowding. Furthermore, we have developed and validated new criteria designed to allow selection using only near-IR and warm Spitzer bands (3.6 and $4.5 \mu \mathrm{m}$ ). These new criteria are important for our follow-up programs on other high-mass star-forming regions that have been observed after Spitzer's liquid helium cryogen supply was exhausted as well 

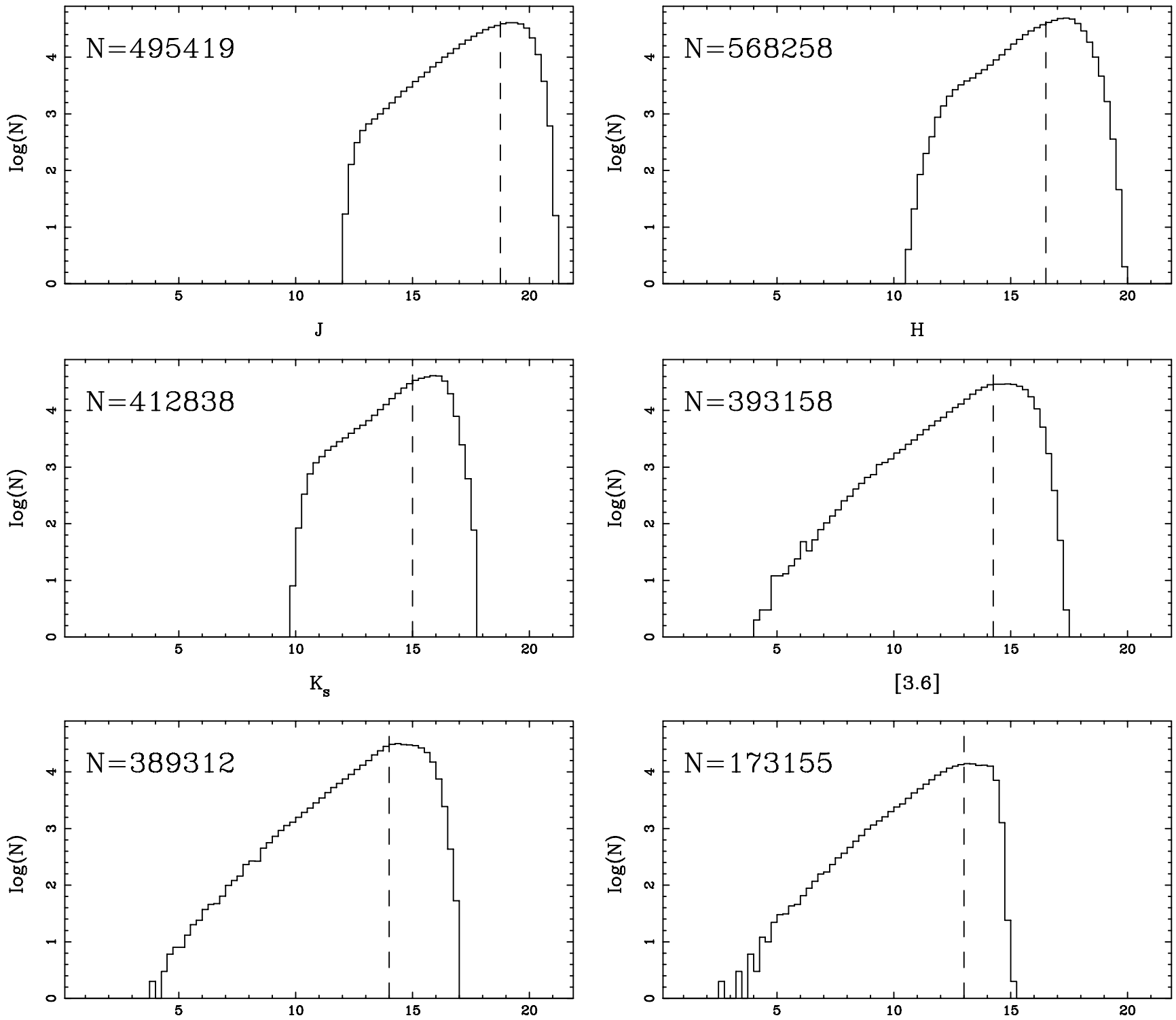

[4.5]
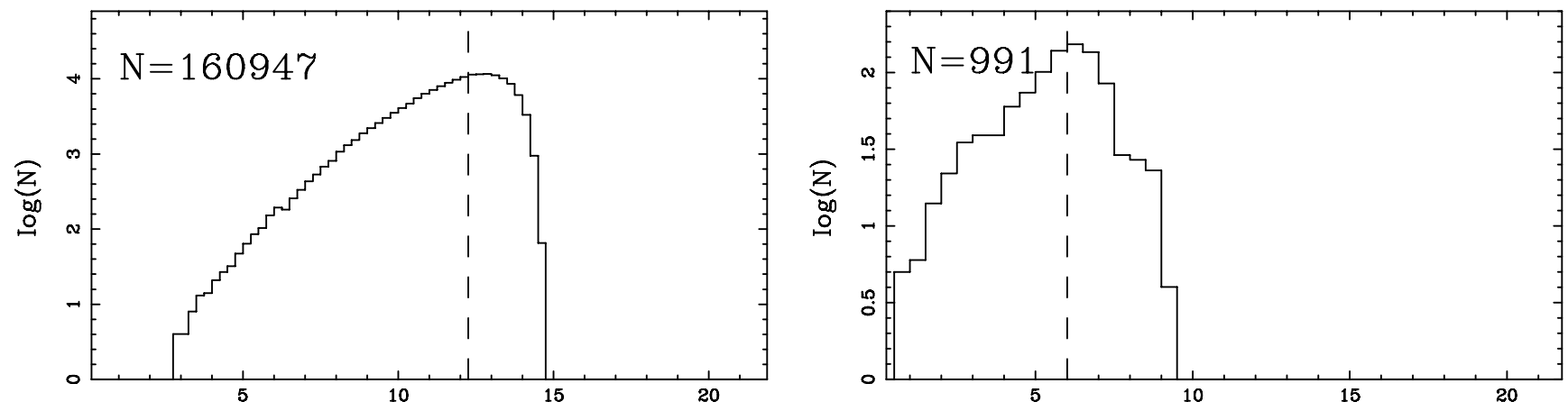

[8.0]

[24.0]

Figure 6. Source Histograms for bands $J$ through $24 \mu \mathrm{m}$ showing the limiting magnitude and completeness limit for each band.

as for other massive regions where existing 5.8 and $8.0 \mu \mathrm{m}$ IRAC observations are compromised by saturation from the very strong polycyclic aromatic hydrocarbon (PAH) extended emission.

To identify the YSOs in NGC 6334, we have first applied the original Gutermuth et al. (2009) criteria to sources with detection at all four IRAC bands, then applied our newly developed criteria to the combined near- and mid-IR point source catalog. We then compare the results of these YSO identification methods to the classification devised by fitting a power law to the SED between 2 and $24 \mu \mathrm{m}$. 
Table 1

NGC 6334 Point Source Catalog

\begin{tabular}{|c|c|c|c|c|c|c|c|c|c|c|c|}
\hline Catalog Entry & R.A. (2000) & Decl. (2000) & $J$ & $H$ & $K_{s}$ & {$[3.6]$} & [4.5] & {$[5.8]$} & {$[8.0]$} & [24.0] & Classification $^{\mathrm{a}}$ \\
\hline 7922 & $17: 17: 57.252$ & $-36: 20: 04.06$ & $13.628 \pm 0.013$ & $12.858 \pm 0.135$ & $10.761 \pm 0.110$ & $6.903 \pm 0.021$ & $6.644 \pm 0.026$ & $6.175 \pm 0.012$ & $5.644 \pm 0.016$ & $3.986 \pm 0.018$ & ICII \\
\hline 24056 & $: 19: 13.033$ & $-36: 20: 26.63$ & $19.421 \pm 0.071$ & $6.508 \pm 0.048$ & $14.389 \pm 0.040$ & $10.444 \pm 0.020$ & $8.723 \pm 0.014$ & $076 \pm 0.015$ & $.948 \pm 0.022$ & $2.302 \pm$ & II \\
\hline 26818 & 7:19:09.717 & $-36: 19: 42.10$ & $19.000 \pm 0.060$ & $4.367 \pm 0.020$ & $11.194 \pm 0.016$ & $7.525 \pm 0.019$ & $6.449 \pm 0.017$ & $5.383 \pm 0.014$ & $4.584 \pm 0.014$ & $.538 \pm 0$. & CI \\
\hline 41234 & $: 19: 11.658$ & $-36: 17: 47.73$ & $15.711 \pm 0.010$ & $12.014 \pm 0.021$ & $11.109 \pm 0.080$ & $7.674 \pm 0.025$ & $6.921 \pm 0.017$ & $6.182 \pm 0.016$ & $.854 \pm 0.0$ & 4.394 & ICI \\
\hline 52364 & $: 18: 03.991$ & $-36: 12: 50.20$ & $13.944 \pm 0.010$ & $12.380 \pm 0.028$ & $11.529 \pm 0.020$ & $10.280 \pm 0.024$ & $9.848 \pm 0.022$ & $9.211 \pm 0.024$ & $8.384=$ & $5.541=$ & ICII \\
\hline 5472 & 7:20:13.901 & $-36: 19: 15.21$ & $15.678 \pm 0.010$ & $12.100 \pm 0.043$ & $11.262 \pm 0.180$ & $8.510 \pm 0.026$ & $8.609 \pm 0.024$ & $8.059 \pm 0.014$ & $8.049 \pm$ & 6.874 & UC \\
\hline 902 & 7:18:00.601 & $-36: 11: 24.54$ & $15.878 \pm 0.009$ & $13.726 \pm 0.006$ & $12.543 \pm 0.010$ & $11.098 \pm 0.024$ & $10.536 \pm 0.025$ & $9.878 \pm 0.026$ & $8.940 \pm 0.020$ & $5.424 \pm$ & ICII \\
\hline 7569 & $17: 17: 56.043$ & $-36: 10: 49.07$ & $14.958 \pm 0.009$ & $12.994 \pm 0.017$ & $11.610 \pm 0.014$ & $9.614 \pm 0.028$ & $8.852 \pm 0.018$ & $7.987 \pm 0.015$ & $7.071 \pm 0.024$ & $3.759 \pm 0$ & ICI \\
\hline 8855 & $17: 20: 28.990$ & $-36: 18: 46.29$ & $16.302 \pm 0.010$ & $12.012 \pm 0.099$ & $10.408 \pm 0.029$ & $8.857 \pm 0.019$ & $8.831 \pm 0.026$ & $8.421 \pm 0.017$ & $8.385 \pm 0.023$ & $6.900 \pm$ & $\mathrm{UC}$ \\
\hline 86342 & $17: 19: 36.644$ & $-36: 14: 22.32$ & $15.793 \pm 0.030$ & $13.446 \pm 0.019$ & $11.572 \pm 0.015$ & $8.763 \pm 0.025$ & $7.904 \pm 0.015$ & $6.862 \pm 0.020$ & $5.360 \pm 0.020$ & $1.908 \pm 0.009$ & ICI \\
\hline 91471 & $17: 19: 07.249$ & $-36: 12: 25.72$ & $16.159 \pm 0.042$ & $13.570 \pm 0.034$ & $12.287 \pm 0.030$ & $10.719 \pm 0.070$ & $10.112 \pm 0.042$ & $9.374 \pm 0.022$ & $8.578 \pm 0.027$ & $4.963 \pm 0.032$ & ICII \\
\hline 95907 & $17: 21: 34.429$ & $-36: 19: 43.48$ & $16.086 \pm 0.010$ & $12.536 \pm 0.039$ & $10.299 \pm 0.021$ & $7.574 \pm 0.022$ & $7.356 \pm 0.016$ & $6.784 \pm 0.012$ & $6.477 \pm 0.022$ & 0.024 & UC \\
\hline 97497 & $17: 20: 06.286$ & $-36: 15: 06.71$ & $15.086 \pm 0.020$ & $13.246 \pm 0.210$ & $11.333 \pm 0.164$ & $7.970 \pm 0.021$ & $8.008 \pm 0.017$ & $7.459 \pm 0.015$ & $7.438 \pm 0.016$ & $.836 \pm 0.068$ & UC \\
\hline 101731 & $17: 22: 00.096$ & $-36: 20: 30.11$ & $15.330 \pm 0.026$ & $12.057 \pm 0.055$ & $10.475 \pm 0.040$ & $9.029 \pm 0.030$ & $9.077 \pm 0.036$ & $8.534 \pm 0.022$ & $8.551 \pm 0.019$ & $6.724 \pm 0.116$ & UC \\
\hline 103075 & $17: 20: 08.806$ & $-36: 14: 41.09$ & $18.888 \pm 0.040$ & $13.611 \pm 0.030$ & $10.647 \pm 0.030$ & $7.852 \pm 0.026$ & $7.596 \pm 0.020$ & $6.922 \pm 0.017$ & $6.658 \pm 0.020$ & $4.541 \pm 0.027$ & ICII \\
\hline 114602 & $17: 21: 01.598$ & $-36: 16: 30.22$ & $16.651 \pm 0.013$ & $14.709 \pm 0.042$ & $13.424 \pm 0.015$ & $11.546 \pm 0.024$ & $11.153 \pm 0.023$ & $10.561 \pm 0.015$ & $9.944 \pm 0.024$ & $7.098 \pm 0.167$ & ICII \\
\hline 115364 & $17: 22: 02.843$ & $-36: 19: 32.04$ & $13.099 \pm 0.114$ & $12.761 \pm 0.121$ & $11.123 \pm 0.080$ & $7.606 \pm 0.024$ & $7.850 \pm 0.017$ & $7.497 \pm 0.022$ & $7.522 \pm 0.022$ & $7.328 \pm 0.204$ & UC \\
\hline 117424 & $17: 21: 16.145$ & $-36: 17: 02.96$ & $17.746 \pm 0.038$ & $15.153 \pm 0.041$ & $13.583 \pm 0.010$ & $10.251 \pm 0.032$ & $9.821 \pm 0.022$ & $9.020 \pm 0.016$ & $8.214 \pm 0.016$ & $4.111 \pm 0.017$ & ICII \\
\hline 117803 & $17: 21: 45.612$ & $-36: 18: 36.68$ & $15.165 \pm 0.010$ & $12.144 \pm 0.047$ & $10.514 \pm 0.020$ & $9.089 \pm 0.027$ & $9.196 \pm 0.023$ & $8.698 \pm 0.014$ & $8.605 \pm 0.017$ & $7.160 \pm 0.175$ & $\mathrm{UC}$ \\
\hline 334274 & $17: 20: 58.800$ & $-36: 15: 10.93$ & $14.737 \pm 0.010$ & $12.844 \pm 0.045$ & $11.835 \pm 0.015$ & $11.140 \pm 0.029$ & $6.658 \pm 0.001$ & $11.112 \pm 0.018$ & $10.938 \pm 0.034$ & & SK \\
\hline 356597 & $17: 21: 34.755$ & $-35: 32: 51.49$ & & $15.858 \pm 0.087$ & $14.831 \pm 0.030$ & $12.137 \pm 0.111$ & $11.241 \pm 0.160$ & $8.904 \pm 0.058$ & $6.874 \pm 0.079$ & & $\mathrm{NCI}$ \\
\hline 368283 & $17: 21: 47.940$ & $-35: 34: 23.59$ & $16.772 \pm 0.010$ & $14.866 \pm 0.026$ & $13.894 \pm 0.011$ & $12.468 \pm 0.023$ & $11.990 \pm 0.033$ & $10.779 \pm 0.050$ & $9.648 \pm 0.104$ & & NCII \\
\hline 370373 & $17: 19: 22.788$ & $-35: 43: 29.94$ & $14.500 \pm 0.010$ & $13.339 \pm 0.019$ & $12.872 \pm 0.020$ & $12.520 \pm 0.026$ & $12.591 \pm 0.026$ & $12.041 \pm 0.048$ & $10.772 \pm 0.089$ & $6.375 \pm 0.099$ & PAHG \\
\hline 489995 & $17: 18: 47.628$ & $-36: 17: 48.77$ & $20.242 \pm 0.099$ & $16.877 \pm 0.023$ & $15.254 \pm 0.020$ & $14.108 \pm 0.038$ & $13.990 \pm 0.039$ & $13.147 \pm 0.088$ & $12.171 \pm 0.135$ & & AGN \\
\hline
\end{tabular}

Notes. ${ }^{\text {a }}$ ICI $=$ IRAC-selected Class I YSO, ICII $=$ IRAC-selected Class II YSO, NCI $=$ near-infrared-selected Class I YSO, NCII $=$ near-infrared-selected Class II YSO, PAHG $=$ PAH galaxy, $\mathrm{AGN}=$ active galactic nuclei, $\mathrm{PAHC}=\mathrm{PAH}$ contaminated aperture, $\mathrm{SK}=$ shock knot, $\mathrm{UC}=$ unclassified source.

(This table is available in its entirety in a machine-readable form in the online journal. A portion is shown here for guidance regarding its form and content.) 

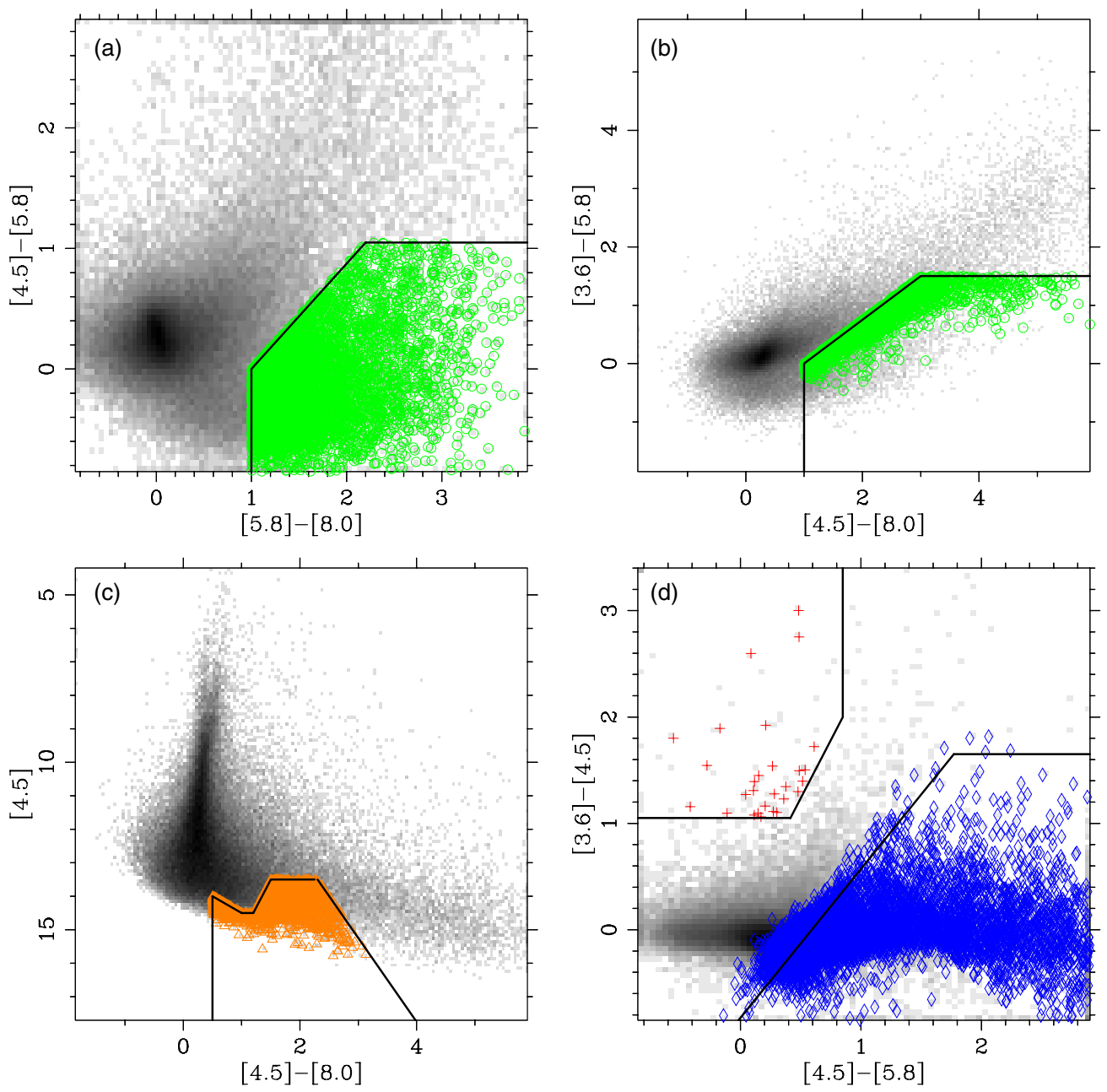

Figure 7. Color-color diagrams used to identify contaminant objects among the sources with detection at all four IRAC bands following the criteria in Gutermuth et al. (2009). The background logarithmic gray-scale indicates the overall source density in each color-color and color-magnitude space. In panels (a) and (b), PAH galaxies are marked with the green circles. In panel (c), candidate AGNs are marked by the orange triangles. Panel (d) shows knots of shocked emission (red plusses) and PAH-contaminated sources (blue diamonds).

(A color version of this figure is available in the online journal.)

Table 2

Excerpt NGC 6334 Point Source Catalog Summary

\begin{tabular}{lccc}
\hline \hline Band & Number of Sources & Magnitude Complete & Limiting Magnitude \\
\hline$J$ & 495,419 & 18.75 & 21.0 \\
$H$ & 568,258 & 16.5 & 19.5 \\
$K_{S}$ & 412,838 & 15.0 & 17.5 \\
$3.6 \mu \mathrm{m}$ & 393,158 & 14.25 & 17.5 \\
$4.5 \mu \mathrm{m}$ & 389,312 & 14.0 & 16.5 \\
$5.8 \mu \mathrm{m}$ & 173,155 & 13.0 & 15.5 \\
$8.0 \mu \mathrm{m}$ & 160,947 & 12.5 & 14.5 \\
$24.0 \mu \mathrm{m}$ & 991 & 6.0 & 9.2 \\
\hline
\end{tabular}

\subsection{IRAC 4 Band Detected Sources}

The first stage of our YSO identification followed the procedure outlined in Gutermuth et al. (2009). This method looks at a variety of color-color and color-magnitude relations for sources detected in all four IRAC bands. First, this method identifies non-YSOs that share some similar color spaces to YSOs and that could contaminate the final source statistics if not removed. These possible contaminants include active galactic nuclei, quasars, resolved knots of shocked gas emission, and faint stellar point sources where the longer wavelength photometry is contaminated by PAH emission, which is a particularly common cause of contamination in this massive starforming region.

We applied this classification scheme to the approximately 80,000 point sources in our catalog with $5 \sigma$ detection at all four IRAC bands. We found that 10,327 sources were likely background galaxies, 41 sources were likely knots of shocked gas emission, and 13,791 sources were identified as faint sources with PAH-contaminated apertures at long wavelengths. After the decontamination process, we were left with 260 sources matching the color selection criteria for Class I YSOs, and 915 sources were identified as Class II YSOs. The application of each step of the full Gutermuth contaminant object identification criteria is shown in Figure 7 and the identified YSOs are shown in Figure 8.

\subsection{Near-IR and 3.6 and $4.5 \mu \mathrm{m}$ Sources}

The SED of a YSO is characterized by excess emission above expected photospheric levels in the IR, increasing toward longer wavelengths through the near- and mid-IR. However, in regions where massive star formation is ongoing, the extreme levels of ionizing radiation from the massive $\mathrm{O}$ and $\mathrm{B}$ stars excites widespread emission from PAH compounds embedded within 


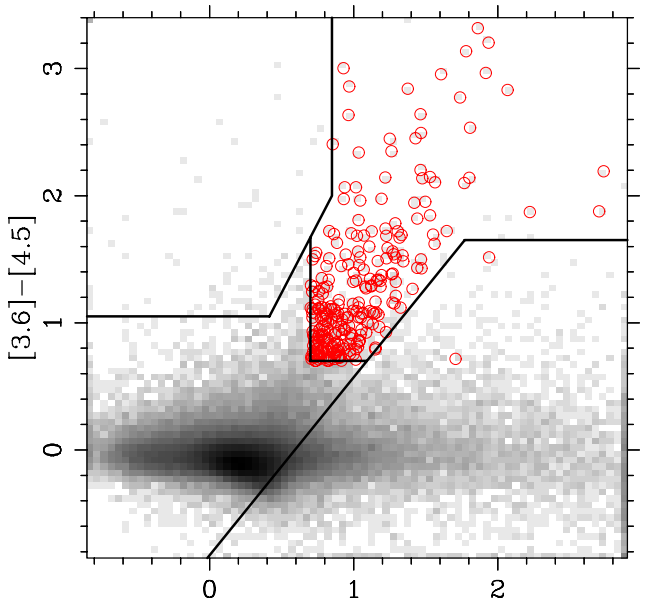

[4.5]-[5.8]

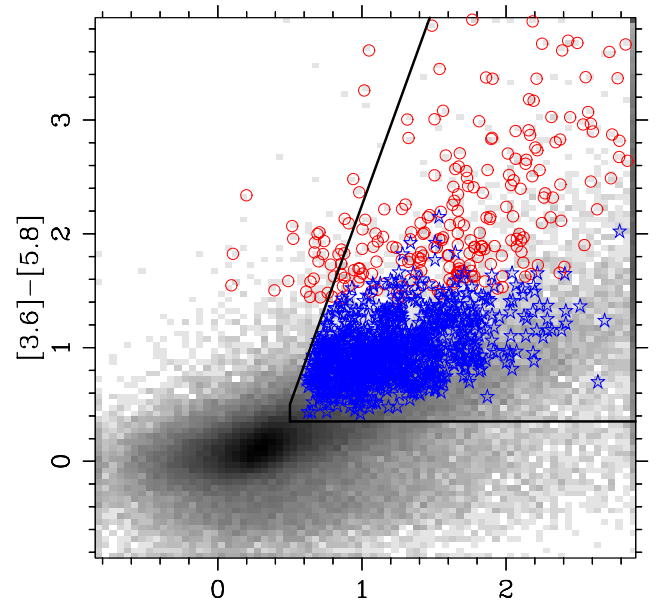

$[4.5]-[8.0]$

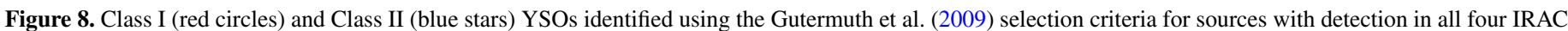

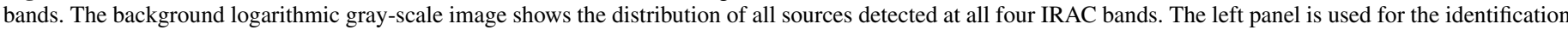
of Class I YSOs. Sources in the region outlined in the right panel not previously identified as Class I YSOs or contaminant objects are identified as Class II YSOs.

(A color version of this figure is available in the online journal.)
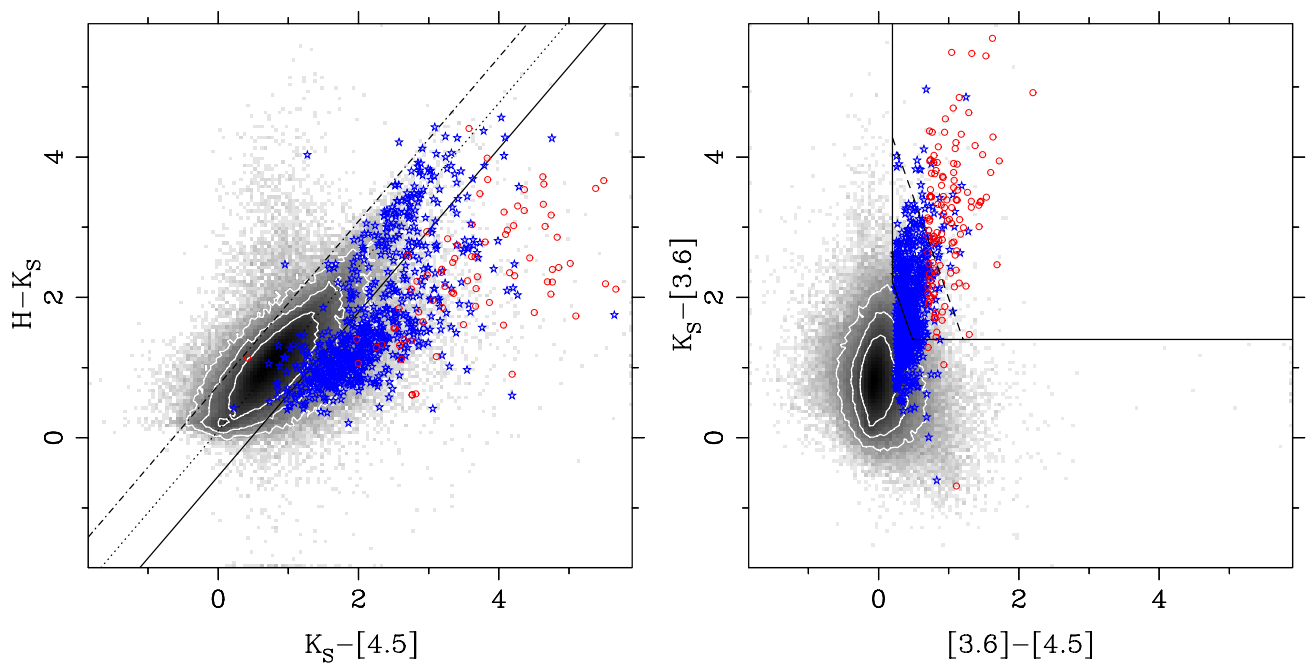

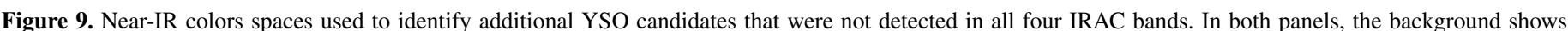

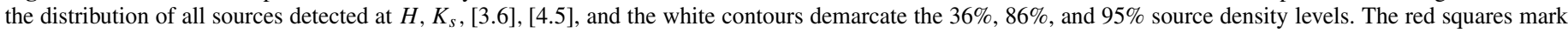

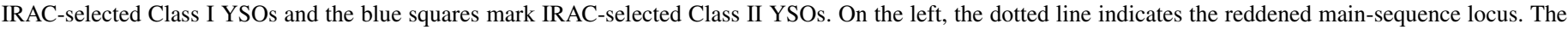

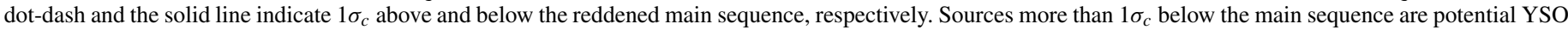

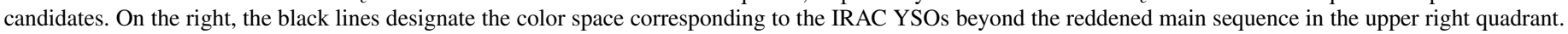
The dark dashed line in the right-hand panel marks the cutoff we have adopted in the near-IR for Class I versus Class II YSOs.

(A color version of this figure is available in the online journal.)

the dust clouds. This extended emission dominates the observed emission at IRAC's 5.8 and $8.0 \mu \mathrm{m}$ bands, drowning out the individual point sources nestled within these structures and saturating the detector in even modestly long integration times. For the second stage of our YSO selection process we identified additional YSO sources that lacked detection at 5.8 or $8.0 \mu \mathrm{m}$ and were thus missed by the Gutermuth et al. (2009) criteria. We used the combined NEWFIRM and IRAC near-IR catalogs for the over 260,000 sources detected at $H, K_{s}, 3.6 \mu \mathrm{m}$, and $4.5 \mu \mathrm{m}$. As mentioned before, these same criteria are also suitable for YSO selection in fields observed during the Spitzer warm mission.

For this stage we first identified the locus of main-sequence stars (assuming an average spectral type of K0) in the $K_{s}-$ [3.6] versus [3.6] - [4.5] and $H-K_{s}$ versus $K_{s}-$ [4.5] color spaces subjected to interstellar reddening. We adopted the parameter $\sigma_{c}$, defined as the maximum error in color for two sources each having the maximum catalog cutoff photometric error of $0.217 \mathrm{mag}(\mathrm{S} / \mathrm{N} \sim 5)$, or $\sigma_{c}=0.307$. Sources falling within $\sigma_{c}$ of the main-sequence reddening vector were considered to be reddened field stars.

We also considered where in these color spaces the sources already identified as YSOs based on the Gutermuth et al. (2009) four IRAC band classification would fall, and the nearIR $K_{s}-$ [3.6] versus [3.6] - [4.5] criteria also presented in Gutermuth et al. (2009). We identify the region beyond the identified $3 \sigma_{c}$ reddened main-sequence star locus and bounding the IRAC-selected YSOs as containing the YSO candidate stars. The equations below describe the bounding regions containing YSO candidate stars and are shown in Figure 9:

$$
[3.6]-[4.5]>0.2
$$



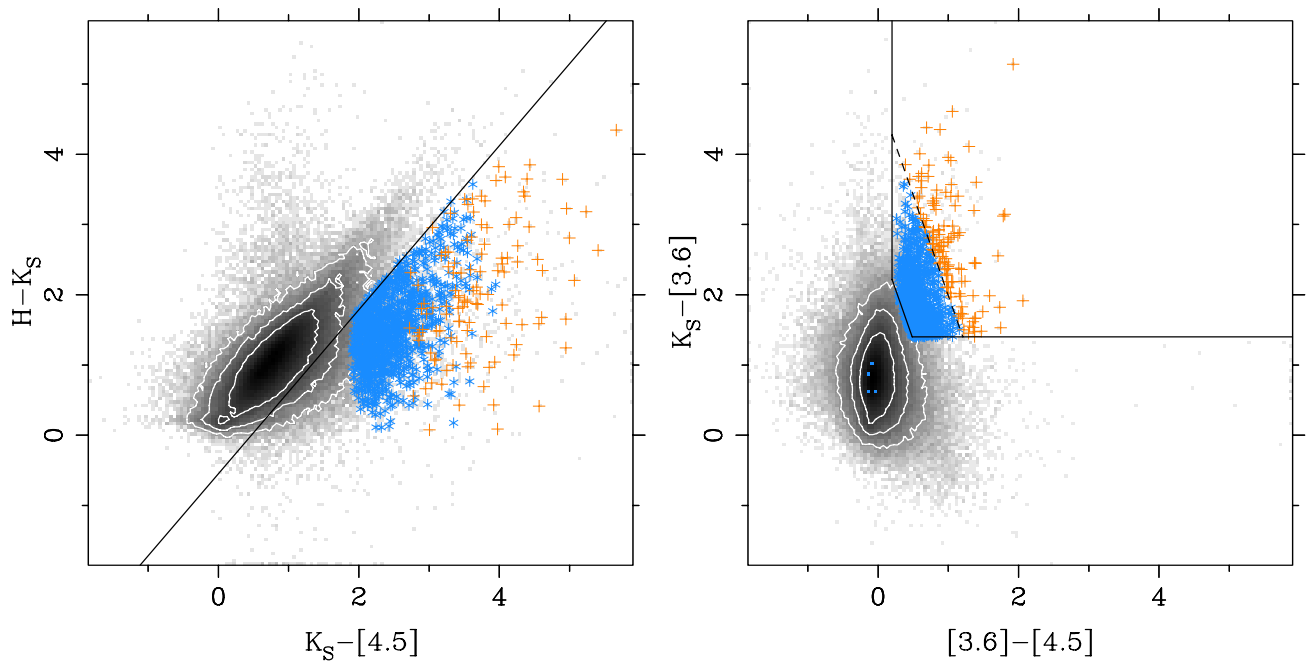

Figure 10. YSO candidates that are identified by their near-IR colors. Gray-scale and contours are the same as in Figure 9. Newly identified YSOs are marked as plusses, Class I YSOs in orange, and Class II YSOs in cyan.

(A color version of this figure is available in the online journal.)

$$
\begin{gathered}
K_{s}-[3.6]>1.4 \\
K_{s}-[3.6]>-2.857([3.6]-[4.5]-0.101)+2.5 \\
H-K_{s}<1.168\left(K_{s}-[4.5]\right)-0.526 .
\end{gathered}
$$

Sources that matched all of the above criteria are considered YSO candidates and are shown in Figure 10. Sources with extremely red colors matching the constraints below are further identified as candidate Class I YSOs, while the rest that only meet the first set of near-IR criteria are identified as Class II YSO candidates:

$$
\begin{gathered}
K_{s}-[3.6]>-2.857([3.6]-[4.5]-0.401)+3.7 \\
K_{s}-[4.5]>2 .
\end{gathered}
$$

Using these criteria, 115 Class I candidates and 993 Class II candidate YSOs are added to the previously identified (four IRAC bands) YSOs. The spatial distribution of the YSO candidate sources throughout the field can be seen in Figures 11 and 12. Viewed in projection against the cloud, the location of Class I sources is strongly correlated to the high density regions, which show up both as the infrared dark clouds (IRDCs) and the bright filaments in the $8 \mu \mathrm{m}$ IRAC image. The Class II sources are also strongly clustered, although a larger number of them are found further from the filamentary structure of NGC 6334. Although some additional spread in Class II location may be expected due to their expected more advanced age, this may also be an indication of the higher contamination fraction present in the Class II population.

Overall, the near-IR cuts are somewhat more restrictive than the selection requiring four IRAC bands. This decreases the amount of contamination from sources extinct only by interstellar and not circumstellar material. In Figure 9, a significant number of IRAC-identified Class II YSOs can be seen within the color spaces belonging to reddened field stars. In total, 81 (31\%) IRAC-selected Class I YSOs and 707 (78\%) IRAC-selected Class II YSOs are also detected at both $H$ and $K_{s}$ bands. Within this sample, $6 \%$ of Class I YSO candidates do not

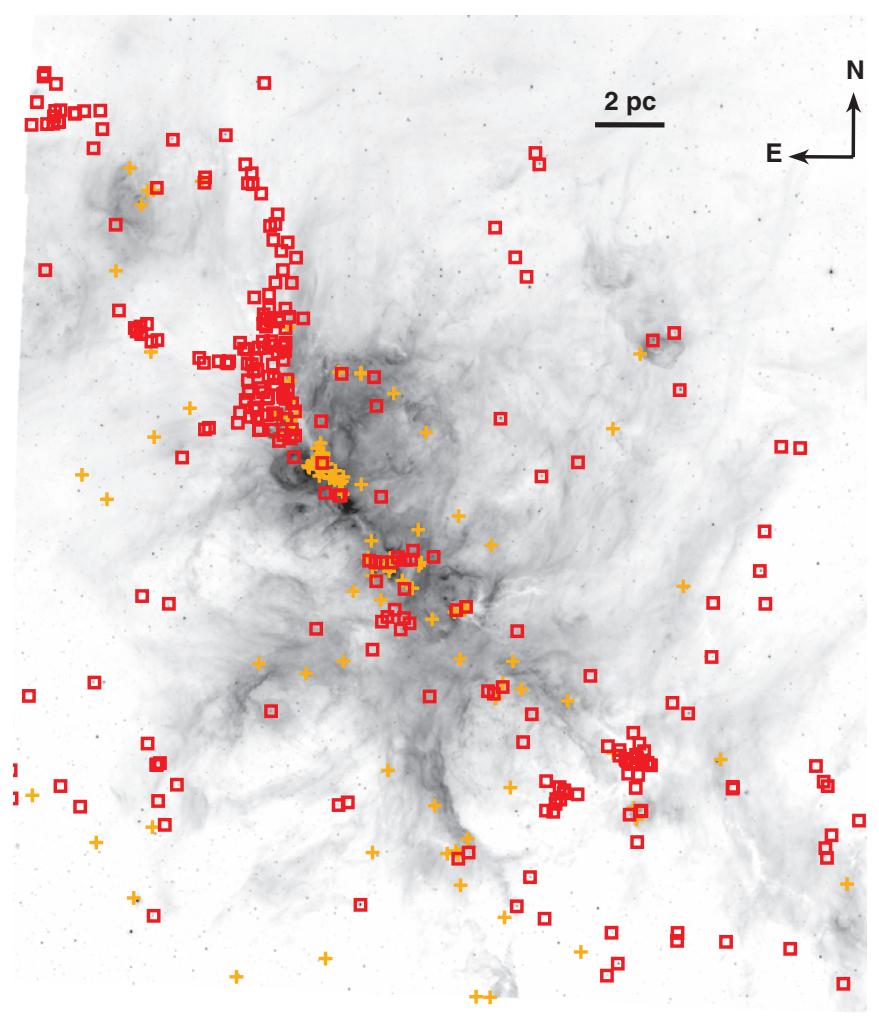

Figure 11. Spatial distribution of all the Class I YSO candidates plotted over the IRAC $8.0 \mu \mathrm{m}$ emission. The IRAC-selected candidates are plotted as red squares and the candidates added using the near-IR criteria are shown as orange plusses. Many Class I sources in the bright nebulous regions of NGC 6334 that saturated at long IRAC observations are recovered by the near-IR criteria.

(A color version of this figure is available in the online journal.)

meet the adopted near-IR criteria and $62 \%$ of the IRAC-selected Class II YSO candidates fail at least one of the near-IR YSO criteria. These sources are within $3 \sigma$ of the reddened mainsequence locus and many are co-aligned with high extinction features seen in the derived NGC 6334 extinction map (see Section 5.1). These sources have a high probability to be reddened sources, either Class III YSOs/pre-main-sequence stars associated with NGC 6334 or unrelated background Galactic stars. We have not attempted a detailed characterization of the 


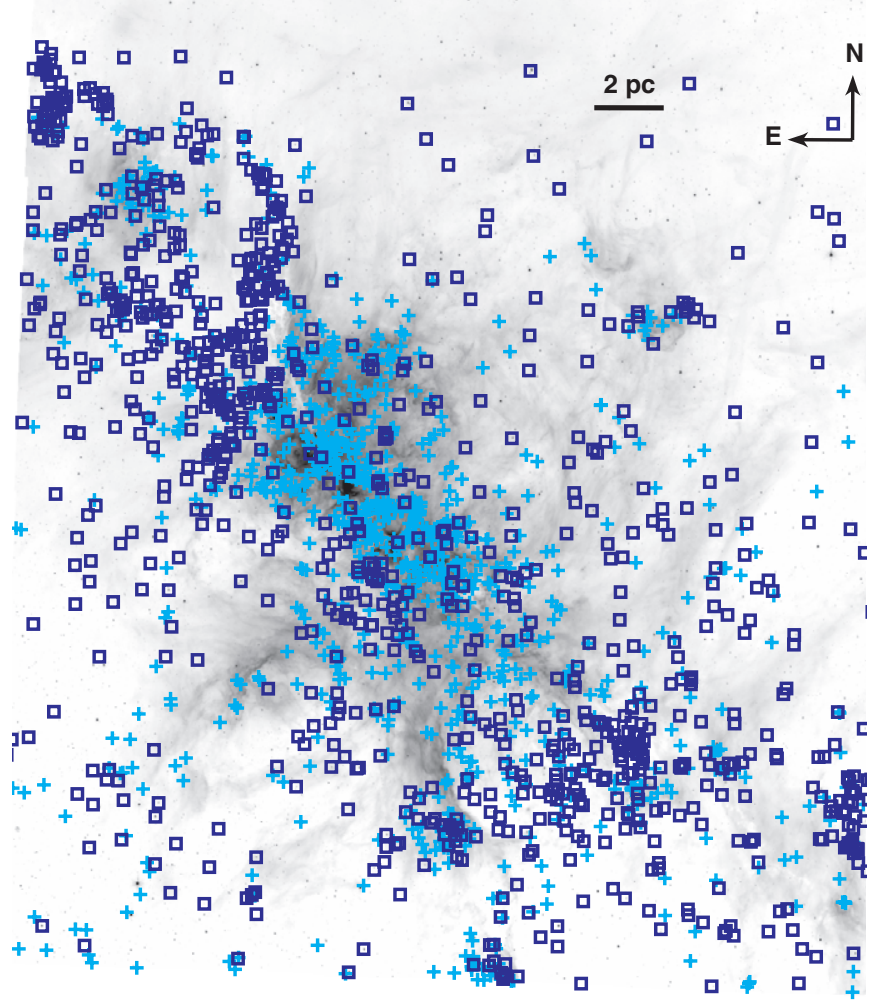

Figure 12. Spatial distribution of all the Class II YSO candidates plotted over the IRAC $8.0 \mu \mathrm{m}$ emission. The IRAC-selected candidates are plotted as blue squares and the candidates added using the near-IR criteria are shown as cyan plusses. The additional Class II sources identified by the near-IR criteria display show a more strongly clustered distribution than the IRAC four band sources, a consequence of the stricter criteria employed for near-IR YSO selection.

(A color version of this figure is available in the online journal.)

Class III/pre-main-sequence population in this work due to the difficulty inherent in distinguishing a small physical IR excess from the scatter due to photometric error and matching between different beam sizes in this crowded field.

Due to the slight differences in the near-IR and IRAC four bands YSO identification methods in the overlapping $3.6-4.5 \mu \mathrm{m}$ region, there is some mismatching between clas-

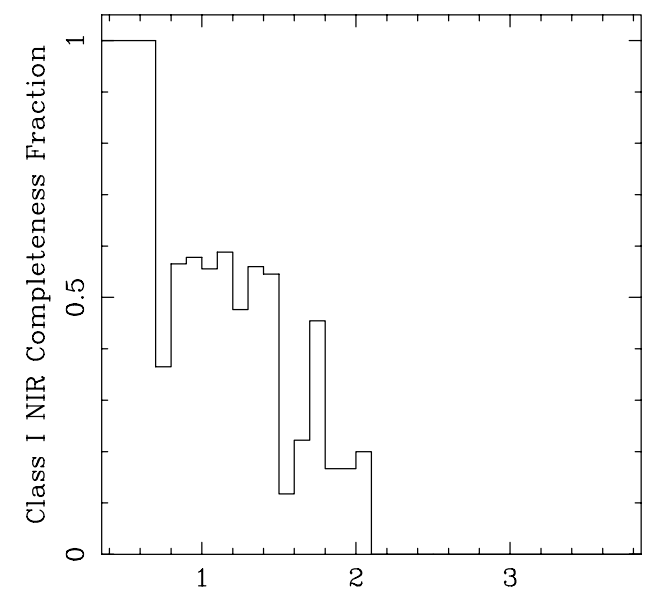

[3.6]-[4.5]
Table 3

YSO Candidates Identified Using Color Criteria

\begin{tabular}{lcr}
\hline \hline Required Bands & Class I & Class II \\
\hline$[3.6],[4.5],[5.8],[8.0]$ & 260 & 915 \\
$H, K_{s},[3.6],[4.5]$ & 115 & 993 \\
Total & 375 & 1908 \\
\hline
\end{tabular}

sification schemes. Of the 81 IRAC-selected Class I YSOs that were detected at both $H$ and $K_{s}, 24(30 \%)$ are identified by the near-IR criteria as Class II sources. Of the 707 IRAC-selected Class II YSOs detected at $H$ and $K_{s}, 18(2.5 \%)$ are identified by the near-IR criteria as Class I sources. For consistency, we retain the IRAC four bands classification for all these sources.

Combined, our near-IR and mid-IR color criteria identified 375 Class I YSOs and 1908 Class II YSOs associated with NGC 6334. The completeness of the near-IR criteria depends on the color of the source, with the fraction of YSOs recovered decreasing toward redder colors. Figure 13 shows the distribution in [3.6] - [4.5] for the sources that are classified by both the IRAC four band and near-IR classification schemes as the same YSO class. It is apparent that the near-IR classification misses out on the reddest sources, which are too deeply embedded to be detected at $H$ or even $K_{s}$. The downturn of the Class II source distribution below [3.6] $-[4.5]<0.5$ also suggests that the near-IR criteria are successfully selecting against lightly reddened sources where the majority of contaminants would fall.

We can use NGC 6334 as a test case to estimate the fraction of the YSO population our near-IR criteria will be able to recover when applied to other massive star-forming regions. If we apply only our near-IR selection cuts to NGC 6334, we identify 1602 ( $84 \%$ of the 1908 total) Class II YSOs. Of the 375 Class I YSOs, our near-IR selection cuts identify 189 (50\%), biased toward detecting the more evolved (less-red) sources. The statistics of the color-identified YSO candidates are summarized in Table 3.

\subsection{SED Slope Comparison}

We have also examined the class distribution of YSOs determined by fitting the slope of the SED in the mid-IR. The class identification for the slope of $\log \left(\lambda F_{\lambda}\right)$ versus $\log (\lambda)$

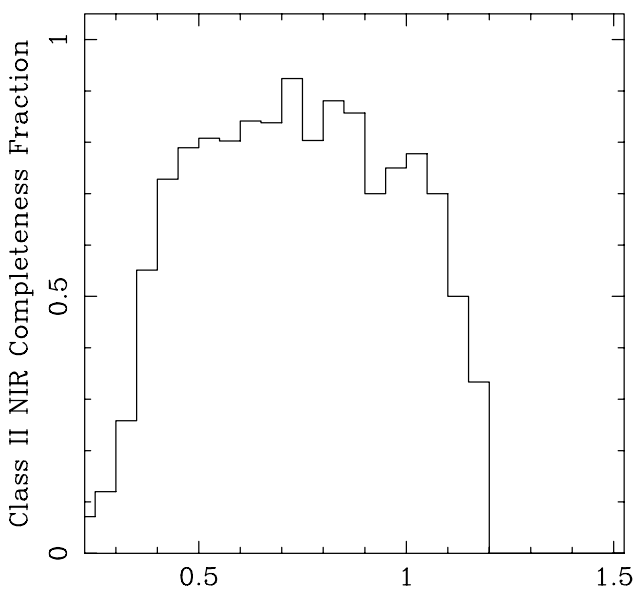

[3.6]-[4.5]

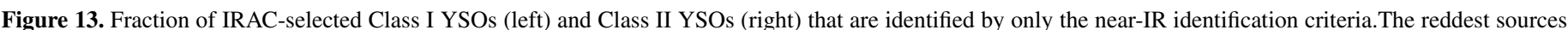

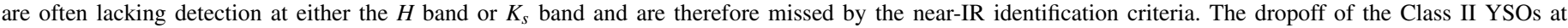

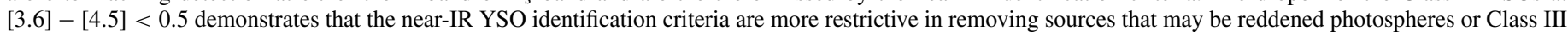
YSOs. 

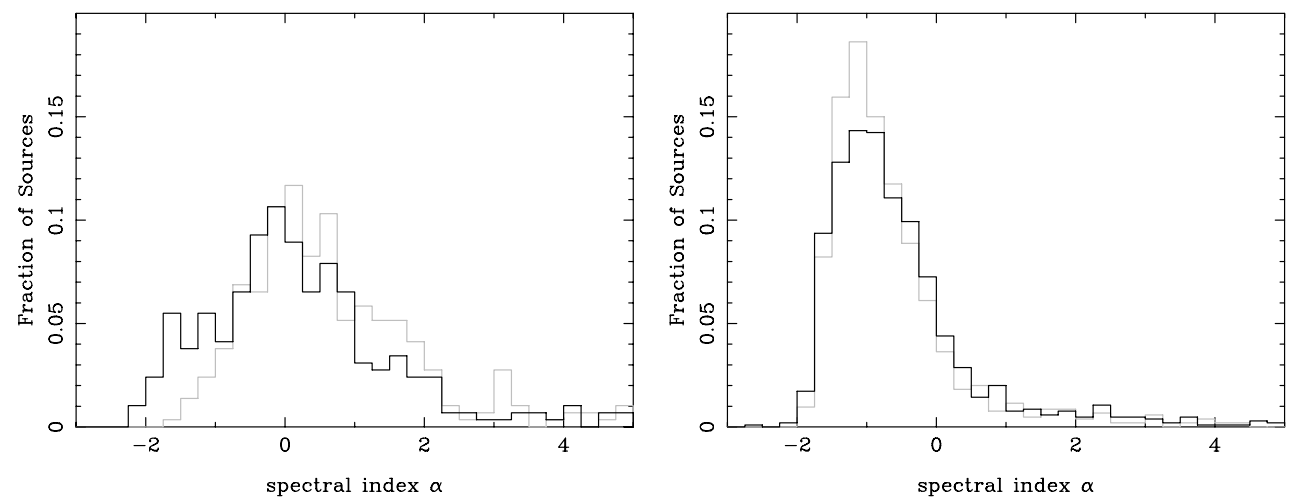

Figure 14. Distribution of SED spectral index $\alpha$ for sources identified as YSO candidates using the color criteria outlined in Section 4 . The left panel shows Class I sources and the right panel shows Class II sources. The slope is determined by fitting the SED for at least three bands starting from $K_{s}$ band (gray/narrow line) or $4.5 \mu \mathrm{m}$ (black/thick line) through $24.0 \mu \mathrm{m}$.

Table 4

SED Based YSO Classification for YSO Candidates Identified by Color Selection

\begin{tabular}{lccccc}
\hline \hline Shortest Included Wavelength & Total Sources & Class I & Flat Spectrum & Class II & Class III \\
\hline$K_{S}$ & 2283 & 697 & 525 & 934 & 127 \\
& & $30.5 \%$ & $23.0 \%$ & $40.9 \%$ & $5.6 \%$ \\
$4.5 \mu \mathrm{m}$ & 1338 & 260 & 223 & 761 & 94 \\
& & $19.4 \%$ & $16.7 \%$ & $56.9 \%$ & $7.0 \%$ \\
\hline
\end{tabular}

between 2 and $\sim 20 \mu \mathrm{m}$ from Lada (1987) is

$$
\alpha \geqslant 0.3 \text { Class I }
$$

$0.3>\alpha \geqslant-0.3$ Flat Spectrum

$$
\begin{aligned}
& -0.3>\alpha \geqslant-1.6 \text { Class II } \\
& -1.6>\alpha \geqslant-2.7 \text { Class III. }
\end{aligned}
$$

To determine the intrinsic slope of the YSO's SED (spectral index $\alpha$ ), we should first de-redden the observations to remove the effect of line-of-sight material. However, it is difficult to gauge how deeply embedded a source is within the molecular cloud. Using the value in the extinction map gives the best guess for the extinction if the source was located on the far side of the molecular cloud, which could result in serious overcorrection for many sources. The effect of the interstellar extinction is most prominent at short wavelength, e.g., $K_{s}$ band and shorter in this survey. We have chosen not to deredden these sources, and instead we compare how the YSO class distribution changes when we only fit the slope of the SED at longer wavelengths. We compare the distribution of $\alpha$ for the sources fit from the $K_{s}$ band and longward compared to fitting only from $4.5 \mu \mathrm{m}$ and longer, similar to the methods used in Megeath et al. (2009) and Kryukova et al. (2012).

Table 4 shows the distribution of the 2283 color-identified YSO candidates by class as given by the spectral index $\alpha$. Figure 14 shows how the distribution of the spectral index $\alpha$ differs when using a minimum wavelength of $K_{s}$ versus $4.5 \mu \mathrm{m}$ to fit the power law to the SED. When we use the $K_{s}$ band as the minimum wavelength to fit the spectral index $\alpha$, we see a shift in the distribution toward a greater number of Class I/Flat Spectrum sources. This likely corresponds to the difficult to separate population of Class II YSOs that are more deeply embedded in the cloud, and thus seen behind a larger amount of interstellar extinction. Source SED classification that relies solely on $\lambda \geqslant 4.5 \mu \mathrm{m}$ is therefore less sensitive to the effects of extinction and provides a superior estimate of evolutionary state. Even when fitting the SED from $4.5 \mu \mathrm{m}$ longward, we see a number of sources identified as Class I YSOs based on the color-color diagrams that appear more likely to be Class II YSOs based on the full slope of the SED. As we will discuss in Section 5.1, the observed area of NGC 6334 contains a significant amount of high column density material that could contribute to cross-contamination of Class I YSOs by reddened Class II YSOs. Even using the power-law fit from $4.5 \mu \mathrm{m}$ longward, the vast majority of color-identified YSO candidates meet the criteria to be either Class I or Class II YSOs, and all have $\alpha \geqslant-2.7$ indicating excess emission at IR wavelengths indicative of circumstellar material. Table 5 lists all the YSO sources identified by the IRAC and near-IR based color selection criteria and gives $\alpha$ fit from the $K_{s}$ band longward.

\subsection{Contamination by Foreground and Background Sources in Warm Spitzer Data}

Misidentifying contaminant objects as YSOs remains an issue for photometry-based identification methods. Although the Gutermuth et al. criteria are very useful for identifying potential contaminant objects, they require detection at 5.8 or $8.0 \mu \mathrm{m}$. Now that Spitzer has exhausted its cryogen and is operating in the warm mission, new IRAC observations of star-forming regions are only able to obtain photometry at 3.6 and $4.5 \mu \mathrm{m}$. We have used our near-IR YSO identification criteria, defined in Section 4.2, to quantify the amount of contamination that we would have seen in our YSO population if our observations for this region had been limited to only warm mission data. If we applied only the near-IR criteria to our full point source catalog, we would identify 1579 sources as YSO candidates: 189 Class I YSOs (50\% complete) and 1602 Class II YSOs ( $84 \%$ complete).

Background galaxies are one class of potential contaminant objects. Near-IR galaxy counts from Martini (2001) estimate that a survey with our sensitivity would detect approximately $1.3 \times 10^{4}$ galaxies per $\mathrm{deg}^{2}$, consistent with the 10,327 suspected extragalactic sources in our catalog identified by the Gutermuth 
Excerpt from NGC 6334 Young Stellar Objects Catalog

\begin{tabular}{|c|c|c|c|c|c|c|c|c|c|c|c|c|}
\hline Catalog Entry & R.A. (2000) & Decl. (2000) & $J$ & $H$ & $K_{s}$ & [3.6] & {$[4.5]$} & {$[5.8]$} & {$[8.0]$} & [24.0] & Classification $^{\mathrm{a}}$ & $\alpha^{\mathrm{b}}$ \\
\hline 315684 & 17:18:06.496 & $-36: 10: 25.90$ & $18.398 \pm 0.020$ & $17.064 \pm 0.028$ & $15.870 \pm 0.030$ & $13.905 \pm 0.032$ & $13.466 \pm 0.029$ & $13.445 \pm 0.129$ & $\operatorname{nan} \pm$ nan & $\operatorname{nan} \pm$ nan & NIRCII & -1.709 \\
\hline 315692 & 17:21:41.687 & $-36: 08: 58.60$ & $18.946 \pm 0.050$ & $17.222 \pm 0.049$ & $16.037 \pm 0.035$ & $14.438 \pm 0.120$ & $13.761 \pm 0.099$ & nan \pm nan & $11.777 \pm 0.104$ & $\operatorname{nan} \pm$ nan & NIRCII & 0.284 \\
\hline 315739 & $17: 18: 25.276$ & $-35: 52: 59.87$ & $\operatorname{nan} \pm$ nan & $\operatorname{nan} \pm$ nan & $\operatorname{nan} \pm$ nan & $8.333 \pm 0.015$ & $7.589 \pm 0.018$ & $6.826 \pm 0.015$ & $6.484 \pm 0.034$ & $4.938 \pm 0.029$ & ICI & -1.320 \\
\hline 315741 & 17:21:05.007 & $-35: 45: 40.96$ & $\operatorname{nan} \pm$ nan & nan \pm nan & $16.361 \pm 0.053$ & $13.367 \pm 0.029$ & $12.155 \pm 0.023$ & $10.862 \pm 0.020$ & $9.972 \pm 0.036$ & $\operatorname{nan} \pm$ nan & ICI & 1.218 \\
\hline 315757 & 17:20:17.905 & $-36: 01: 25.02$ & $16.458 \pm 0.010$ & $14.574 \pm 0.020$ & $13.637 \pm 0.020$ & $12.437 \pm 0.048$ & $12.158 \pm 0.052$ & $11.793 \pm 0.061$ & $11 \pm 0.087$ & nan \pm nan & ICII & -1.242 \\
\hline 315774 & 17:21:18.731 & $-35: 43: 11.47$ & $\operatorname{nan} \pm$ nan & $18.574 \pm 0.111$ & $16.747 \pm 0.109$ & $14.332 \pm 0.103$ & $13.867 \pm 0.113$ & $\operatorname{nan} \pm$ nan & $\operatorname{nan} \pm$ nan & $\operatorname{nan} \pm$ nan & NIRCII & 0.984 \\
\hline 315779 & 17:19:08.870 & $-35: 57: 49.40$ & $15.911 \pm 0.010$ & $14.460 \pm 0.016$ & $13.564 \pm 0.020$ & $12.375 \pm 0.039$ & $12.058 \pm 0.039$ & $11.73 \pm 0.023$ & $11.057 \pm 0.028$ & $\operatorname{nan} \pm$ nan & ICII & -1.312 \\
\hline 315783 & 17:20:55.782 & $-36: 04: 07.13$ & $18.488 \pm 0.070$ & $15.649 \pm 0.030$ & $13.455 \pm 0.020$ & $9.42 \pm 0.018$ & $7.936 \pm 0.020$ & $6.591 \pm 0.014$ & $5.561 \pm 0.015$ & $\operatorname{nan} \pm$ nan & ICI & 1.612 \\
\hline 315805 & 17:20:25.945 & $-35: 50: 47.92$ & $\operatorname{nan} \pm$ nan & $17.258 \pm 0.030$ & $14.586 \pm 0.020$ & $12.043 \pm 0.047$ & $11.5 \pm 0.021$ & $\operatorname{nan} \pm$ nan & nan \pm nan & $\operatorname{nan} \pm$ nan & NIRCII & 1.169 \\
\hline 315808 & 17:22:08.859 & $-35: 41: 17.49$ & $14.302 \pm 0.007$ & $12.926 \pm 0.020$ & $12.048 \pm 0.020$ & $10.457 \pm 0.035$ & $10.134 \pm 0.025$ & $9.769 \pm 0.030$ & $9.399 \pm 0.035$ & $\operatorname{nan} \pm$ nan & ICII & -1.601 \\
\hline 315819 & $17: 21: 09.213$ & $-35: 49: 28.93$ & $20.375 \pm 0.139$ & $14.849 \pm 0.010$ & $11.899 \pm 0.020$ & $9.677 \pm 0.020$ & $9.176 \pm 0.016$ & $8.618 \pm 0.015$ & $8.481 \pm 0.016$ & nan \pm nan & ICII & -1.439 \\
\hline 315820 & $17: 19: 26.314$ & $-36: 10: 16.40$ & $\operatorname{nan} \pm$ nan & $17.370 \pm 0.030$ & $14.530 \pm 0.020$ & $12.811 \pm 0.033$ & $12.494 \pm 0.031$ & $12.092 \pm 0.039$ & $11.282 \pm 0.085$ & nan \pm nan & ICII & -1.176 \\
\hline 315826 & 17:20:22.109 & $-35: 50: 53.84$ & $17.830 \pm 0.020$ & $15.214 \pm 0.020$ & $13.088 \pm 0.020$ & $10.129 \pm 0.023$ & $9.412 \pm 0.017$ & $8.547 \pm 0.012$ & $7.559 \pm 0.029$ & $\operatorname{nan} \pm$ nan & ICI & 0.176 \\
\hline 315827 & $17: 20: 23.518$ & $-35: 45: 17.24$ & $\operatorname{nan} \pm$ nan & $\operatorname{nan} \pm$ nan & $\operatorname{nan} \pm \operatorname{nan}$ & $14.709 \pm 0.155$ & $12.837 \pm 0.137$ & $10.614 \pm 0.100$ & $8.29 \pm 0.121$ & $\operatorname{nan} \pm$ nan & ICI & 4.658 \\
\hline 315842 & 17:19:13.351 & $-36: 09: 16.10$ & $\operatorname{nan} \pm$ nan & $16.673 \pm 0.030$ & $12.755 \pm 0.019$ & $10.146 \pm 0.026$ & $9.909 \pm 0.018$ & $9.309 \pm 0.022$ & $9.234 \pm 0.018$ & nan \pm nan & ICII & -1.710 \\
\hline 315845 & 17:22:04.564 & $-36: 19: 52.04$ & $19.029 \pm 0.047$ & $17.134 \pm 0.080$ & $16.525 \pm 0.130$ & $15.069 \pm 0.185$ & $14.431 \pm 0.132$ & $\operatorname{nan} \pm$ nan & $\operatorname{nan} \pm$ nan & nan \pm nan & NIRCII & -0.143 \\
\hline 315880 & 17:19:04.841 & $-36: 10: 05.06$ & $19.437 \pm 0.100$ & $17.934 \pm 0.060$ & $15.730 \pm 0.039$ & $11.352 \pm 0.082$ & $10.662 \pm 0.053$ & $8.329 \pm 0.094$ & $8.348 \pm 0.158$ & $\operatorname{nan} \pm$ nan & NIRCI & 1.389 \\
\hline 315896 & 17:21:24.899 & $-35: 44: 22.73$ & $18.423 \pm 0.030$ & $15.640 \pm 0.020$ & $14.319 \pm 0.020$ & $13.484 \pm 0.033$ & $13.162 \pm 0.033$ & $12.898 \pm 0.095$ & $12.186 \pm 0.162$ & $\operatorname{nan} \pm$ nan & ICII & -1.469 \\
\hline 315897 & 17:19:04.386 & $-36: 09: 24.02$ & $\operatorname{nan} \pm$ nan & $18.807 \pm 0.135$ & $16.510 \pm 0.079$ & $14.225 \pm 0.052$ & $13.928 \pm 0.046$ & $\operatorname{nan} \pm$ nan & $\operatorname{nan} \pm$ nan & $\operatorname{nan} \pm$ nan & NIRCII & 0.506 \\
\hline
\end{tabular}

Notes.

${ }^{\mathrm{a}}$ ICI $=$ IRAC-selected Class I YSO, ICII = IRAC-selected Class II YSO, NCI = Near-infrared-selected Class I YSO, NCII $=$ Near-infrared-selected Class II YSO

b $\alpha$ fit from $K_{s}$ band longward.

(This table is available in its entirety in a machine-readable form in the online journal. A portion is shown here for guidance regarding its form and content.) 
Table 6

Fraction of Galactic and Extragalactic Sources Matching Warm Spitzer YSO Classification Criteria

\begin{tabular}{lcr}
\hline \hline Source Type & Class I & Class II \\
\hline RGB/Early-AGB & $\ldots$ & $\ldots$ \\
AGB & $2.4 \%$ & $5.9 \%$ \\
Post-AGB & $1.3 \%$ & $33.3 \%$ \\
Galaxies & $<0.1 \%$ & $0.6 \%$ \\
\hline
\end{tabular}

et al. (2009) criteria. Of these galaxies identified by their IRAC four band colors, 62 have the correct colors to match our $H, K_{s}$, 3.6 , and $4.5 \mu \mathrm{m}$ YSO color criteria. Only 1 of these 62 sources is red enough to match the Class I criteria; the other 61 sources are only moderately red and would have been identified as Class II YSOs. We therefore would expect that approximately $3.8 \%$ of detected Class II YSO candidates and $0.5 \%$ of detected Class I YSO candidates identified using only near-IR and warm Spitzer color criteria would actually be background galaxies.

The primary culprit for Galactic contamination of our YSO population is asymptotic giant branch (AGB) stars. To asses the level of contamination by evolved stars in our warm Spitzer YSO classification scheme, we referenced a sample of spectroscopically confirmed, intrinsically red post-main-sequence stars in the Large Magellanic Clouds (LMCs), identified by various authors and matched to IRAC and near-IR photometry from the "Surveying the Agents of a Galaxy's Evolution" (SAGE; Meixner et al. 2006) catalog. We used the selection criteria from Section 4 to estimate the fraction of post-main-sequence sources matching our YSO colors. Although none of the sample red giant branch (RGB) and early-AGB stars matched the YSO criteria, we found matches among the more evolved stars.

Our post-main-sequence LMC template sample included 423 AGB stars (from Matsuura et al. 2009 and Kontizas et al. 2001), 267 carbon-rich, and 156 oxygen-rich. Of these, 35 AGB stars passed the selection cuts and were identified as YSO candidates, 10 Class I and 25 Class II. Out of the sample of 75 postAGB stars from van Aarle et al. (2011), 1 matched the Class I YSO criteria and 25 matched the Class II YSO criteria. We would identify $2.4 \%$ of the AGB stars in a field as Class I YSOs and $5.9 \%$ of the field AGB stars as Class II YSOs. One of the previous results from GLIMPSE was a quantification of the Galactic distribution of intrinsically red stellar sources (Robitaille et al. 2008). At $l=351^{\circ}, b=0$.7, Robitaille et al. (2008) determined that there would be approximately 80 AGB sources per square degree; indicating that our $0.8 \mathrm{deg}^{2}$ region, covered from $J$ band through $4.5 \mu \mathrm{m}$, should contain roughly 68 AGB stars. By applying the SAGE-derived contamination fraction estimates, we would have falsely identified two of those AGB stars as Class I YSOs and four of those AGB stars as Class II YSOs. We tabulated the fraction of different types of contaminant sources that have colors compatible to Class I and II YSOs in Table 6. We examined a sample of 75 post-AGB stars (Hughes \& Wood 1990; Pinheiro et al. 2010; Pompéia et al. 2008; Woods et al. 2011). We found only $1.3 \%$ of the post-AGB sources matched our near-IR Class I YSO selection cuts, but fully one-third (33.3\%) matched the colors for Class II YSOs. Fortunately, the post-AGB phase evolves rapidly $\left(<10^{5} \mathrm{yr}\right.$; van Winckel 2003) and they tend to be uniformly distributed on the sky, so the contamination of YSO samples by post-AGB stars within a star-forming region should be negligible. Table 7 lists the number of expected contaminant objects matching YSO colors we would expect per square degree in a survey with our depth.
Table 7

Number of Expected Galactic and Extragalactic Contaminants Per Square Degree

\begin{tabular}{lcc}
\hline \hline Source Type & Class I Contaminants & Class II Contaminants \\
\hline Post-main-sequence stars & 2.7 & 6.4 \\
Galaxies & $\ldots$ & 212.7 \\
\hline
\end{tabular}

Final confirmation of the nature of our YSO candidates would require follow-up spectroscopic observations. Similar follow-up studies in other star-forming regions have recently shown that the contamination fraction of color-selected YSO candidates can be quite high, especially for sources with small and moderate IR excess: for transition disk objects in eight star-forming regions, the observed AGB contamination fraction ranged between 20\% and $100 \%$ (Romero et al. 2012). Their identified transition disk objects all had [3.6] $-[4.5]<0.25$. In our sample of coloridentified Class II YSOs, this cut applies to only 28 sources.

\section{DISCUSSION}

The results of the YSO census for Class I and Class II objects allows us to estimate global properties of the stellar population associated with NGC 6334, such as its rate of formation and the star formation efficiency. By expanding to use the entire point source catalog, we can also map the extinction across the survey area to help determine NGC 6334's cloud structure and mass.

\subsection{Extinction, Column Density, and Total Gas Mass}

The NICER algorithm (Lombardi \& Alves 2001) was applied to the sources in the $0.8 \mathrm{deg}^{2}$ region covered at $J, H, K_{s}, 3.6$, and $4.5 \mu \mathrm{m}$. This method uses the $J-H, H-K_{s}, K_{s}-$ [3.6], and [3.6] - [4.5] near-IR colors to determine the line-of-sight extinction toward each source. The extremely dense conditions in the dark clouds in NGC 6334 result in regions lacking sufficient numbers of stars to accurately determine the true value of the extinction. These extremely dense regions have a lower bound of $A_{V}>30$ and overlap with the dark clouds of the field as seen in all four IRAC bands and even the MIPS $24 \mu$ m images. In these regions we used the local stellar density to derive a lower bound for the total extinction. The un-patched extinction map is shown in Figure 15, with contours of point source density to highlight the areas where the large average distances between point sources suggest the low extinction values in the map are anomalous.

Dust column densities derived from Herschel Hi-GAL (Molinari et al. 2010) and HOBYS (Motte et al. 2010) results show that along the dense ridges in NGC 6334, the total visual extinction exceeds several hundred magnitudes (Russeil et al. 2013). Approximately $10 \mathrm{pc}^{2}$ along the ridge have column densities that exceed the $0.7 \mathrm{~g} \mathrm{~cm}^{-2}\left(A_{V} \sim 200\right)$ suggested as the threshold for massive stars formation by Krumholz \& McKee (2008). The extremely high column densities in the ridge are supported by extinction measurements from Chandra X-ray observations of NGC 6334 (Feigelson et al. 2009).

A recent analysis of the optically visible $\mathrm{O}$ and $\mathrm{B}$ stars in NGC 6334 determined an average line-of-sight extinction of $A_{V}=4.12 \pm 0.20$ (Persi \& Tapia 2008). We subtracted this line-of-sight component from the patched extinction map and summed the extinction over the map, converting from magnitudes of extinction to column density using $N\left(\mathrm{H}_{2}\right)=$ $1.37 \times 10^{21} A_{V}$ (Heiderman et al. 2010; Draine 2003). At the assumed distance to NGC 6334 of $1.6 \mathrm{kpc}$, the $0.75 \mathrm{deg}^{2}$ 


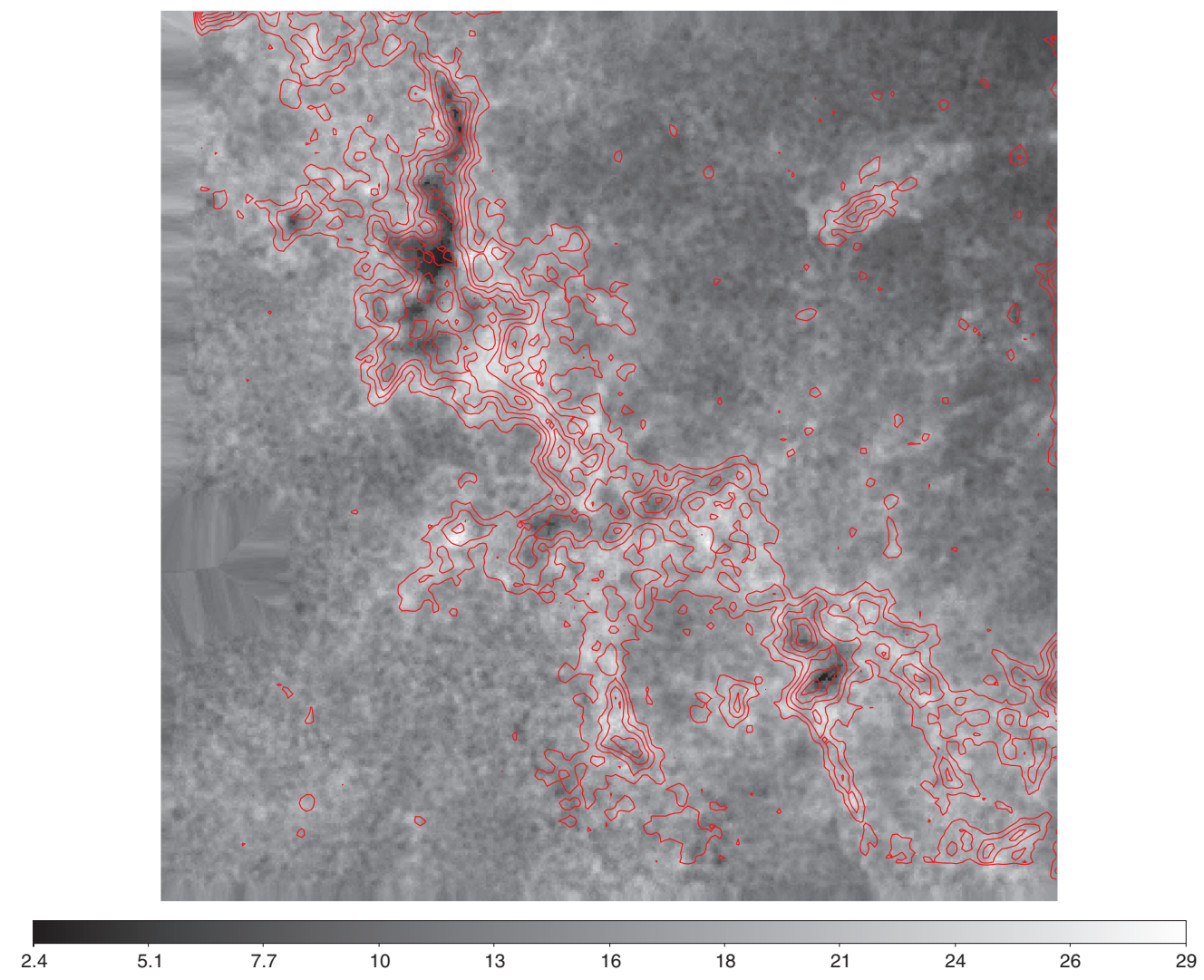

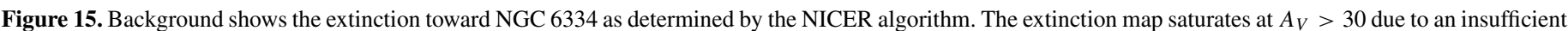

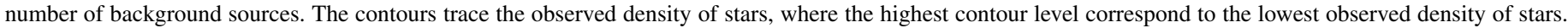

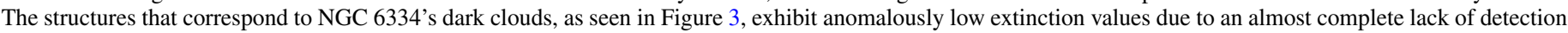
of background sources.

(A color version of this figure is available in the online journal.)

extinction map corresponds to $590 \mathrm{pc}^{2}$. We assume a standard composition of $63 \%$ hydrogen, $36 \%$ helium, and $1 \%$ dust to determine the total cloud mass from the calculated hydrogen column density. We find that the mass of NGC 6334 is $2.2 \times 10^{5} M_{\odot}$. Due to the limitations of calculating column density from extinction when the extinction map saturates at approximately $A_{V} \sim 30$, this mass should be considered a lower limit. Indeed, the HOBYS team reports $3.8 \times 10^{5} M_{\odot}$ for NGC 6334 (Russeil et al. 2013). However, the area surveyed by the HOBYS team also extends significantly farther than our extinction map, which may contribute to their higher mass estimate.

Our mass estimate closely matches the mass derived from the HiGAL column density map pertaining to the same area. After subtracting the line-of-sight component, we find up to $70 \%$ of the mass of cloud appears to be contained in dense material above the general star formation threshold of $A_{V} \sim 10$ (e.g., McKee 1989; Lada et al. 2010; Heiderman et al. 2010). This high-mass concentration is found both in the extinction map derived from NICER as well as the HiGAL column density map.

\subsection{NGC 6334 Star Formation Rate and Efficiency}

In nearby star-forming regions, it is reasonable to assume that the observed sample of YSOs is nearly complete and to assign an average mass to the observed population in order to estimate physical characteristics such as the star formation rate and efficiency. For NGC 6334, due to the increased distance, the implementation of strict criteria to identify the YSOs, and the effects of saturation and decreased efficacy of source detection in the bright nebular emission, the observed Class I and Class II populations are noticeably incomplete. In addition, due to the crowding of this field we have not attempted to isolate the Class III YSO population from the Galactic foreground and background stars in our point source catalog.

We have instead used the observed Class I and Class II sample to estimate the total size of the YSO population in NGC 6334. We have compared our work to the NGC 6334 X-ray point source catalog to estimate the number of young stars in the complex that lack significant IR excess and to meet the YSO identification criteria (Feigelson et al. 2009).

\subsubsection{Estimating Stellar Population Mass}

We have used the observed population of YSO candidates and YSO models to estimate the lowest mass objects at which our survey is complete, and infer the lower-mass population from that point by assuming a standard Kroupa initial mass function (IMF).

First, we took the simulated photometry for the 200,710 models computed by Robitaille et al. (2006) and scaled the observed magnitudes from $1 \mathrm{kpc}$ (the default distance) to the adopted distance to NGC 6334, $1.6 \mathrm{kpc}$. We ran the model photometry through the YSO selection cuts we outlined in Section 4. This enabled us to isolate model YSO SEDs with the same observable properties as the Class I and Class II YSOs that we identified in NGC 6334. Models with relatively high 

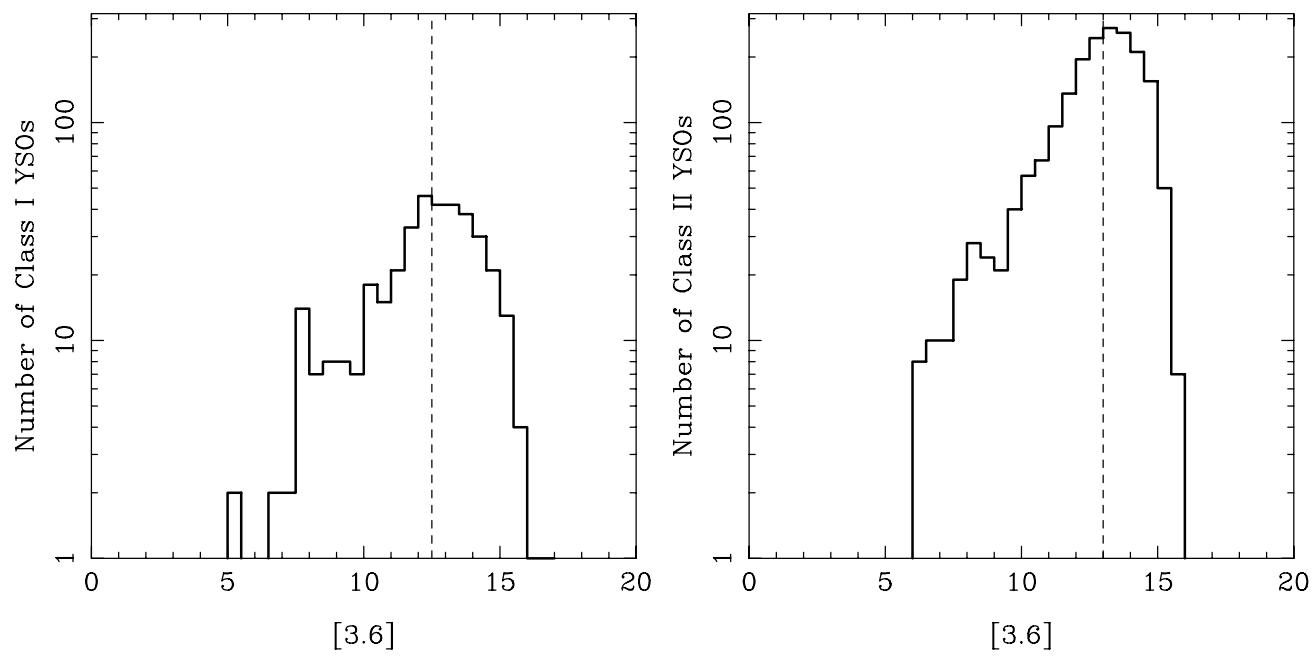

Figure 16. $3.6 \mu \mathrm{m}$ magnitude histograms for the 375 Class I (left panel) and 1908 Class II (right panel) YSO candidates. The dashed lines mark the approximate magnitude at which the YSO census is assumed to be complete for each class, at [3.6] $=12.5$ for Class I YSOs and [3.6] $=13$ for Class II YSOs.

envelope accretion rates were excluded as Class 0 sources that are generally not detected below $10 \mu \mathrm{m}$. After identifying the Class I and Class II YSO models, the leftover models that were not red enough to meet Class I or Class II selection criteria were selected to represent Class III YSOs.

We examined each YSO Class independently and grouped models by their $3.6 \mu \mathrm{m}$ magnitude in bins 0.1 mag wide. In each magnitude bin, we looked at the distribution of stellar mass. We selected the peak of the distribution as the average mass for models within that magnitude range. We integrated under the stellar mass distribution around the peak until our mass range included $65 \%$ of the models. The smallest mass within this range was adopted as the lower bound for the mass, and the highest mass within this range was adopted as the upper bound. Using this approach, we have established an average mass-magnitude relation for the YSO models based on their brightness at $3.6 \mu \mathrm{m}$.

Next, we used the observed $3.6 \mu \mathrm{m}$ magnitude histogram for the NGC 6334 Class I and II YSOs (shown in Figure 16) to determine the approximate magnitude at which each class is complete, identified by the point at which the magnitude histogram ceases to increase. In this way, we determine the Class I YSO population is complete to [3.6] $=12.5$, with 220 YSOs brighter than this limit. The Class II YSO population is complete to [3.6] $=13$, with 987 YSOs brighter than this limit. We used the average mass-magnitude relation derived from the YSO models to translate our magnitudes into a mass range. We find the [3.6] $=12.5$ for Class I YSOs corresponds to $M_{\text {complete }}=0.75_{-0.6}^{+0.9} M_{\odot}$, with a median value of $1.2 M_{\odot}$. For Class II YSOs we find $M_{\text {complete }}=0.3_{-0.15}^{+0.3} M_{\odot}$, with a median value of $0.3 M_{\odot}$, all at the assumed distance of $1.6 \mathrm{kpc}$.

We used the number of YSOs brighter than the completeness limit (220 Class I and 987 Class II) to normalize a Kroupa IMF with $\alpha=2.3$ for $M>0.5 M_{\odot}$ and $\alpha=1.3$ for $M<0.5 M_{\odot}$. By extrapolating from $M_{\text {complete }}$ down to the hydrogen burning limit $\left(0.08 M_{\odot}\right)$, we estimate that there are approximately 1600 Class I YSOs in NGC 6334 and 2400 Class II YSOs. The derived mass in Class I YSOs is $800_{-600}^{+1500} M_{\odot}$. The derived mass in Class II YSOs is $1200_{-500}^{+1400} M_{\odot}$.

Although we did not directly isolate the Class III population in our point source catalog, we can use the fact that young stars have a high level of X-ray activity to characterize the population of the more evolved stars in NGC 6334. A Chandra X-ray survey of NGC 6334 (Feigelson et al. 2009) detected approximately 1600 sources identified as pre-main-sequence stars; we find only 49 Class I and 165 Class II YSOs within 6" of these Xray sources, confirming that the majority (almost 90\%) of the $\mathrm{X}$-ray sources are likely to be more evolved Class III YSOs/premain-sequence stars that are otherwise not included in our YSO census. Feigelson et al. (2009) determined that the X-ray sample was complete to approximately $1 M_{\odot}$, and that extrapolating to low X-ray levels below their detection threshold would suggest a total of 20,000-30,000 pre-main-sequence stars associated with the complex.

We matched our IRAC sources detected at $3.6 \mu \mathrm{m}$ to the Chandra X-ray catalog, finding 1400 total matches and adopt this as our Class III YSO population. Many of these sources display minimal IR excess, with a median [3.6] - [4.5] of 0.09. As seen in Figure 17, the Class III sources are complete to $[3.6]=13$, with 873 sources brighter than this limit. From the Robitaille models, for Class III YSOs [3.6] = 13 corresponds to approximately $M_{\text {complete }}=1.5 \pm 0.6 M_{\odot}$ at a distance of $1.6 \mathrm{kpc}$. We apply the same method of normalizing a Kroupa IMF as we applied to the Class I and Class II YSOs. We find the 873 Class III/pre-main-sequence stars detected brighter than [3.6] = 13 represent a total population with mass $7900_{-3900}^{+4700} M_{\odot}$. Using a standard $0.5 M_{\odot}$ average stellar mass for the $20,000-30,000$ stars reported by Feigelson et al. (2009), we would expect 10,000-15,000 $M_{\odot}$ in Class III YSOs in NGC 6334. The high level of diffuse emission that makes it difficult to achieve a uniform completeness across the observed field means that even our YSO samples that are assumed to be complete may be missing a significant number of sources. The true value for the YSO stellar mass is therefore likely to be closer to the upper end of the ranges derived by examining the sources detected at $3.6 \mu \mathrm{m}$. By extension, the derived physical quantities we report (star formation rate and efficiency) may also underrepresent the total star formation activity in NGC 6334 by a factor of up to two.

\subsubsection{Rate and Efficiency of Star Formation in NGC 6334}

The HII regions in NGC 6334 are estimated to be on the order of 2 Myr old or younger (Persi \& Tapia 2008). We adopt 2 Myr as the age of young stars in NGC 6334 and use the total mass in YSOs to determine the overall star formation rate 

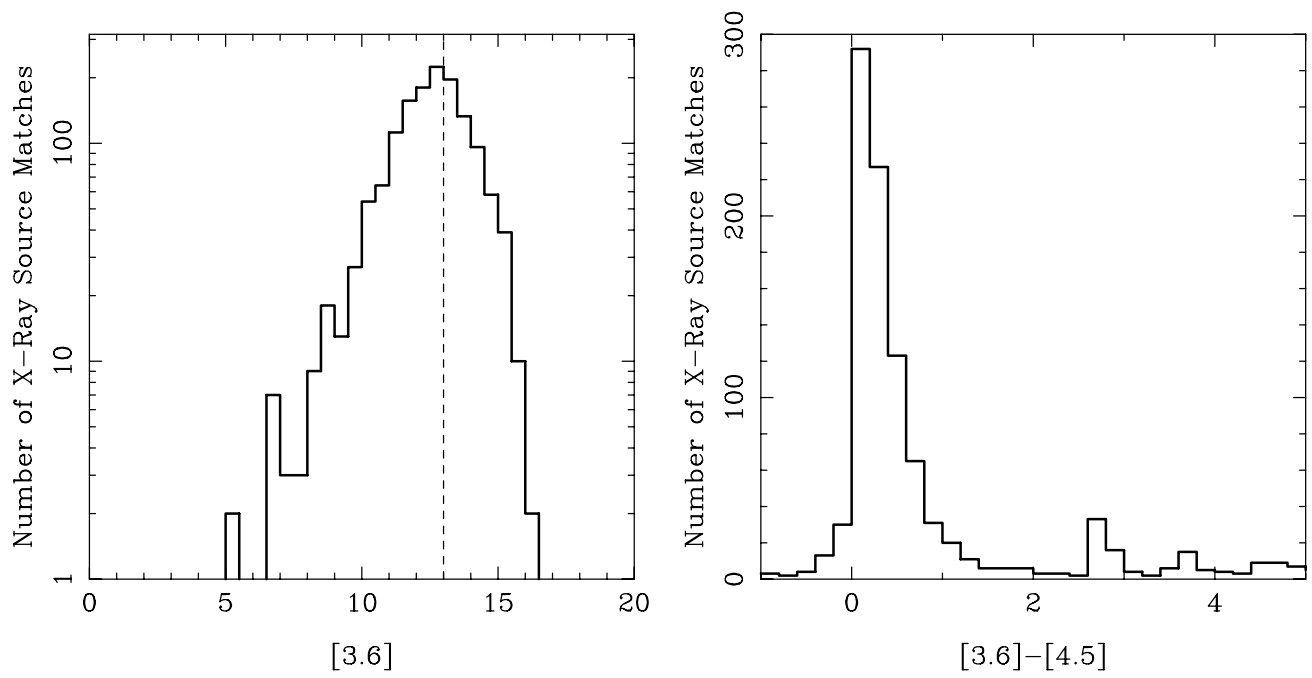

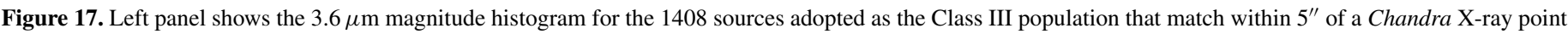

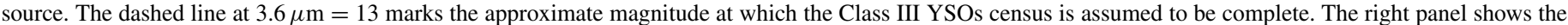
distribution of [3.6] - [4.5] for the 1294 X-ray sources detected in both IRAC bands.

SFR $\sim 4900_{-2500}^{+3800} M_{\odot} \mathrm{Myr}^{-1}$. The $0.8 \mathrm{deg}^{2}$ area used to derive the YSO catalog corresponds to approximately $600 \mathrm{pc}^{2}$ at a distance of $1.6 \mathrm{kpc}$. Over this wide-field view of NGC 6334, we find that the average star formation rate surface density is 8.2 $2_{-4.2}^{+6.3} M_{\odot} \mathrm{Myr}^{-1} \mathrm{pc}^{-2}$.

We use the Class I YSO population mass extrapolated from the observed Class I YSOs to estimate the current star formation rate in NGC 6334. The mean lifetime of Class I YSOs has been estimated to be $0.4 \pm 0.2 \mathrm{Myr}$ in low-mass star-forming regions (Evans et al. 2009), although this is likely to be shorter for more massive stars. The average value of our Class I total mass estimate from Section 5.2.1 is $800 M_{\odot}$. We use the $0.4 \mathrm{Myr}$ lifespan of Class I YSOs and find the current star formation rate in NGC 6334 is $2000_{-1600}^{+3700} M_{\odot} \mathrm{Myr}^{-1}$. In NGC 6334, the Class I YSOs are almost exclusively found in regions with $A_{V}>8$, which accounts for almost $25 \%$ of the area we have observed $\left(150 \mathrm{pc}^{2}\right)$. Within this dense gas the star formation rate surface density is $\Sigma_{\mathrm{SFR}, \mathrm{CI}}=13.0_{-10.4}^{+24.7} M_{\odot} \mathrm{Myr}^{-1} \mathrm{pc}^{-2}$.

We determine the star formation efficiency in NGC 6334 by adding up the mass of the Class I, II, and III YSOs and comparing them to the cloud mass estimate determined from the extinction map. The poorly characterized Class 0 population may be safely ignored in the star formation efficiency computation; due to the relatively short timescales involved in Class 0 evolution, these sources would contribute only a negligible amount to the total stellar mass content of NGC 6334. Overall, this yields an integrated stellar mass content of NGC 6334 of 4800-17400 $M_{\odot}$. Adopting the estimated $2.2 \times 10^{5} M_{\odot}$ gas mass for the cloud from Section 5.1, we estimate the lower limit for the efficiency of star formation activity in NGC 6334 is $\mathrm{SFE} \sim 0.04_{-0.02}^{+0.03}$. If we adopt the Feigelson et al. (2009) estimate for the size of the stellar X-ray population in NGC 6334, the efficiency may exceed the upper limit of the range we found, $\mathrm{SFE} \geqslant 0.10$. Among the dense cores and clumps of the cloud, we find local star formation efficiency upward of 0.3. The local variations in the efficiency of star formation and sites of cluster formation within NGC 6334 will be discussed in a follow-up paper.

The star formation rate and efficiency we present in this paper are derived using $5 \sigma$ as the maximum photometric error in each band for inclusion in our point source catalog. Sources with higher photometric errors intrinsically have less reliable photometry and have a higher probability to be contaminant objects. We examined the number of YSO candidates we would select from our point source catalog if we changed the maximum allowed photometric error. By decreasing the maximum allowed photometric error to $0.108(10 \sigma)$, we would decrease the number of YSO candidates by approximately $25 \%$, from the original 2283 YSO candidates to 1652 YSO candidates. The spatial distribution of the $10 \sigma$ selected YSOs is virtually indistinguishable from the $5 \sigma$ sources. Additionally, despite the decrease in the number of YSO candidates, we see only a small effect on the derived rate and efficiency of star formation. Our method of determining the mass in YSOs relies on the assumption that our observations are only complete down to a limited magnitude/mass range, and most of the sources observed with photometric errors between $5 \sigma$ and $10 \sigma$ fall below the completeness limit. If we exclude all sources between $5 \sigma$ and $10 \sigma$ and calculate the star formation rate surface density, we would obtain $\Sigma_{\mathrm{SFR}, 10 \sigma}=7.8_{-4.3}^{+5.1} M_{\odot} \mathrm{Myr}^{-1} \mathrm{pc}^{-2}$, a difference of less than $10 \%$ from the value calculated on the full set of YSO candidates detected at $5 \sigma$ or greater.

\subsubsection{Comparing NGC 6334 to Orion and Low-mass Star-forming Regions}

To verify the star formation rates calculated from this method, we have applied our method of determining the star formation rate to the YSO population detected in Orion. The Spitzer Orion survey observed a $572 \mathrm{pc}^{2}$ region and identified a total of 3479 YSOs (Megeath et al. 2012). In Orion, the YSO candidates were identified using similar color-color diagrams and then the YSO candidates were primarily classified based on the slope of their SEDs. Megeath et al. (2012) report 488 likely protostars, and the rest are considered stars with disks. Gutermuth et al. (2011) reports the mass within the surveyed area of Orion is $3.3 \times 10^{4}$. The total mass of Orion A and Orion B is $\sim 2 \times 10^{5} M_{\odot}$ (Bally 2008), similar to the mass we have estimated for NGC 6334. This similarity makes Orion a good benchmark for comparison for NGC 6334.

First, we assume that the sources classified as protostars in Orion are roughly the same class as our Class I YSO population in NGC 6334, and that the stars with disks in Orion are an 

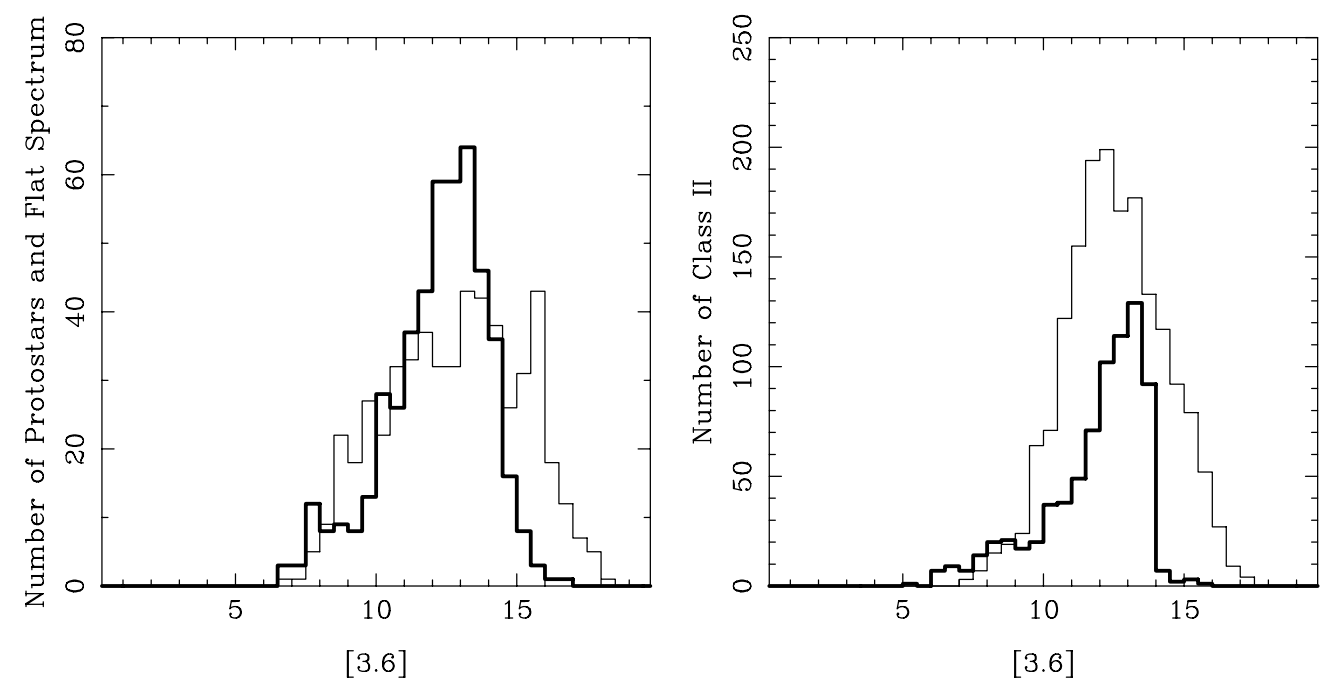

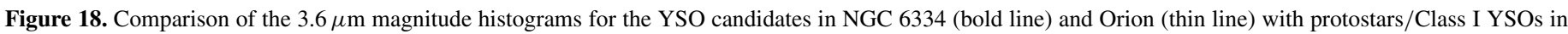

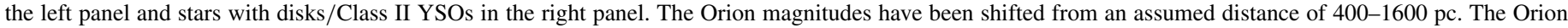

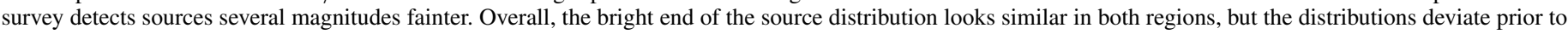
the adopted completeness limit of [3.6] $=12.5$ for the rising/flat spectrum protostars or for [3.6] $=13$ for the Class II YSOs.

equivalent class to the Class II YSOs in NGC 6334. We estimate the Orion YSO population is complete to approximately [3.6] $=14\left(M_{\text {complete }}=0.2 \pm 0.16\right)$ for protostars and [3.6] $=$ $11\left(M_{\text {complete }}=0.3 \pm 0.4\right)$ for stars with disks. Following the method described above, this leads to a total mass in protostars of $320_{-150}^{+220} M_{\odot}$ and $1540_{-1040}^{+770} M_{\odot}$ for stars with disks. Adopting the estimated 2 Myr age suggested for the Orion YSO population gives a total star formation rate $930_{-600}^{+500} M_{\odot} \mathrm{Myr}^{-1}$, or a star formation rate surface density of $1.6_{-1.0}^{+0.9} M_{\odot} \mathrm{Myr}^{-1} \mathrm{pc}^{-2}$. If we follow the standard assumptions for low-mass star-forming regions and apply them to Orion, we adopt a $0.5 M_{\odot}$ average mass and a 2 million year star formation time scale and obtain $\mathrm{SFR}=870 M_{\odot} \mathrm{Myr}^{-1}$, within $10 \%$ of the rate obtained using our method.

Despite the similar masses of the two clouds, the star formation rate in Orion is almost a factor of four smaller than the star formation rate we find in NGC 6334. One possible explanation for this is the difference in dense gas in the two clouds. In NGC 6334, we found that up to $70 \%$ of the mass of the cloud is contained in dense gas above $A_{V}>8$. In Orion, this value is reported to be closer to $10 \%$ (Lada et al. 2009). The much larger reservoir of star-forming gas suggests that NGC 6334's increased star formation rate will continue. It is important to remember that the completeness of NGC 6334's observed YSO population may be even lower than predicted here in this study.

In Figure 18, we compare the $3.6 \mu \mathrm{m}$ magnitude histograms of sources in NGC 6334 and Orion with the same spectral index $\alpha$. We find that the distribution of protostars and flat spectrum sources in NGC 6334 is similar on the bright end. However, the Spitzer Orion survey extends to fainter sources as the difference in distance between the two regions would suggest. For stars with disks/Class II YSOs $(-0.3>\alpha>-1.6)$ we find substantial differences between the Orion and NGC 6334 YSO $3.6 \mu \mathrm{m}$ magnitude histograms. This difference could be attributed to intrinsic differences between the stellar populations forming in the two regions-i.e., a different shape of IMF in one region versus another, or a different history of star formation between NGC 6334 and Orion. If we assume that the overall populations are intrinsically similar, we find that
NGC 6334 is missing a substantial number of Class II sources as bright as $[3.6]=10$. This could partially be due to our more restrictive selection criteria that aim to minimize the number of contaminant objects in our YSO sample. Another issue is the wide-spread extremely bright extended PAH emission in NGC 6334. Earlier Spitzer studies (e.g., Chavarría et al. 2008; Koenig et al. 2008) found that the IRAC sensitivity to YSOs could decrease by up to $3 \mathrm{mag}$ at $8.0 \mu \mathrm{m}$ in fields with bright $\mathrm{PAH}$ emission. For this additional reason, we interpret the star formation rate and efficiency we have calculated for NGC 6334 as a conservative estimate.

Recent results for a sample of 20 local low-mass star-forming regions found that their star formation rate surface densities are between 0.1 and $3.4 M_{\odot} \mathrm{Myr}^{-1} \mathrm{pc}^{-2}$ with an overall average of $1.2 \pm 0.2 M_{\odot} \mathrm{Myr}^{-1} \mathrm{pc}^{-2}$ (Heiderman et al. 2010), which is at least five times lower than NGC 6334. Heiderman et al. also provided an estimate of the rate of ongoing star formation in these same regions through their study of how the star formation rate density varied with increasing gas density in the clouds. Within the $A_{V} \sim 8$ contour, the star formation rate density derived from Class I YSOs in the low-mass cloud sample is $0.03-1.3 \mathrm{M}_{\odot} \mathrm{Myr}^{-1} \mathrm{pc}^{-2}$ with an average of $0.5 M_{\odot} \mathrm{Myr}^{-1} \mathrm{pc}^{-2}$, compared to the $13.0_{-10.4}^{+24.7} M_{\odot} \mathrm{Myr}^{-1} \mathrm{pc}^{-2}$ in NGC 6334.

The term "mini-starburst" has been used to describe very active massive star-forming regions where conditions are similar to those observed in starburst galaxies (Motte et al. 2003). The suggested criteria for a "mini-starburst" are $\Sigma_{\mathrm{SFR}} \sim$ 10-100 $M_{\odot} \mathrm{Myr}^{-1} \mathrm{pc}^{-2}$ and SFE $\sim 0.15$ (Motte et al. 2012). In the IRDC G035.39-00.33 these conditions are met over an approximately $8 \mathrm{pc}^{2}$ region, comparable in size to the dense ridge in NGC 6334. These conditions are found on a wider scale in the massive Galactic star-forming region W43 located at the intersection of the Galactic bar and the Scutum-Centaurus arm. The (conservative) estimate $8.2_{-4.2}^{+6.3}$ for the global star formation rate we present in NGC 6334 is approaching the limit defining mini-starburst conditions. These conditions are fully met when we confine ourselves to the Class I YSOs located within $A_{V}>8$, where we also expect the efficiency to increase, as has been found in other star-forming regions (Gutermuth et al. 2011). 


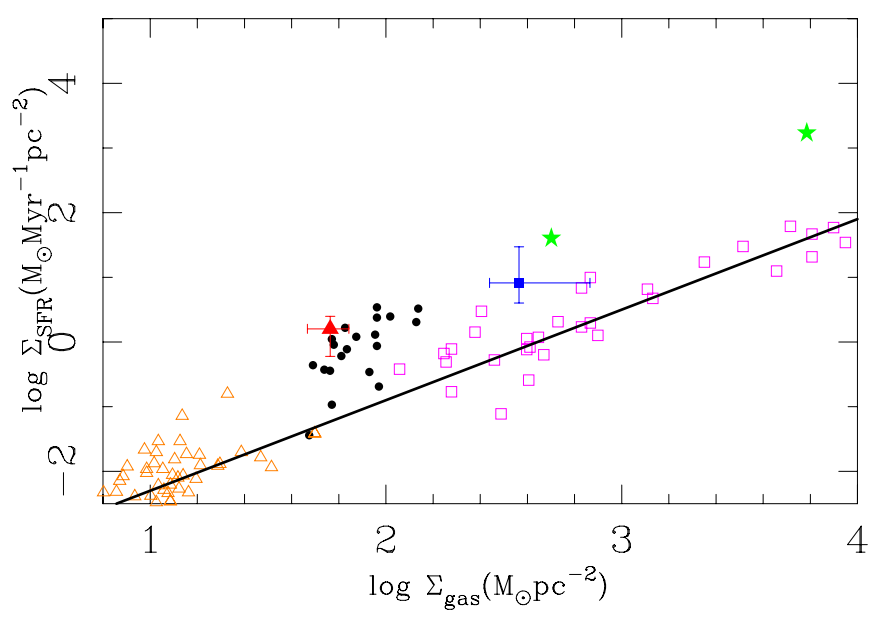

Figure 19. Kennicutt-Schmidt relation comparing the observed star formation rate surface density and gas mass surface density. NGC 6334 is the blue box and the error bars correspond to the uncertainty in the mass estimates of the YSO population and the molecular cloud. Orion is shown as a red filled triangle with corresponding errors bars at the star formation rate surface density derived with our method. Low-mass star-forming regions from Spitzer's c2d and GBS programs are plotted as filled black circles. W43 and G035.39, two Galactic massive star-forming regions identified as prototypical "mini-starbursts" (Motte et al. 2012), are plotted as green stars. The open triangles show a sample of normal spiral galaxies and the open boxes show starburst galaxies. The line marks the Kennicutt-Schmidt relation with $\alpha=1.4$. The vertical offset between the extragalactic and the Galactic star-forming regions likely results from the beam of extragalactic observations being diluted by non-star-forming gas.

(A color version of this figure is available in the online journal.)

This suggests NGC 6334 may also belong to the "mini-starburst" class of star-forming regions.

NGC 6334 does not appear to have any special Galactic location to explain the abundance of dense gas and the subsequent extreme star formation activity taking place within this region. Low-resolution observations of transitions of $\mathrm{CO},{ }^{13} \mathrm{CO}, \mathrm{CS}$, and $\mathrm{NH}_{3}$ found $\mathrm{a} \sim 10 \mathrm{~km} \mathrm{~s}^{-1}$ velocity gradient along the main ridge indicative of a dynamic process such as large-scale infall (Kraemer \& Jackson 1999). A large-scale infall or dynamic merger of multiple filamentary structures could help explain the origin of the high fraction of dense molecular gas in NGC 6334, and in turn the high star formation activity. Recent work attributes the velocity gradient along this ridge to global gravitational collapse of the NGC 6334 molecular cloud (Zernickel et al. 2013).

Figure 19 compares NGC 6334 to other Galactic star-forming regions observed by Spitzer during the Gould Belt Survey (GBS; Harvey et al. 2008; Kirk et al. 2009; Peterson et al. 2011; Spezzi et al. 2011; Hatchell et al. 2012), the Molecular Cores to Planet-forming Disks project (c2d; Evans et al. (2009)), and the prototypical "mini-starburst" regions W43 and G035 from (Motte et al. 2012). The range shown for NGC 6334 reflects the uncertainty in both the mass of the total cloud and the total age and mass of the YSO population. NGC 6334 falls between the low-mass Galactic star-forming regions at the lower left and the Galactic "mini-starburst" regions at the upper right. The location of NGC 6334 and the Galactic "mini-starburst" regions makes it unlikely that the vertical offset between the Galactic star-forming regions and other galaxies in the Kennicutt-Schmidt diagram is due to fundamental differences between regions that are forming high and low-mass stars. Instead, the high star formation rate found in NGC 6334 supports the idea that the primary predictor of a high star formation rate is a large amount of dense gas, as suggested in, e.g., Gutermuth et al. (2011) and Lada et al. (2010), with different dependencies on the density of the star-forming gas. In a typical galaxy, the total gas mass is diluted and only a small fraction is found in molecular clouds dense enough to participate in star formation activity.

\section{SUMMARY}

We have obtained deep observations of NGC 6334 in the near- and mid-IR, cataloging $>700,000$ Galactic point sources in a $0.8 \mathrm{deg}^{2}$ region. Based on their near- and mid-IR colors, 2283 of these have been identified as Class I or Class II YSOs belonging to the NGC 6334 star-forming complex. Overall, the observed YSO population is found to be complete to just below $1 M_{\odot}$. We have modified existing YSO identification schemes in order to identify the population of YSOs in other star-forming regions where long wavelength IRAC data is not available, as well as estimate the contamination, completeness, and reliability of those measurements. The full point source catalog was used to map the extinction across NGC 6334 to estimate the region's mass and from that the efficiency of star formation. The $0.8 \mathrm{deg}^{2}$ area used to derive the YSO catalog corresponds to approximately $600 \mathrm{pc}^{2}$ at a distance of $1.6 \mathrm{kpc}$. Over this wide-field view of NGC 6334, we find that the average star formation rate surface density is $8.2_{-4.2}^{+6.3} M_{\odot} \mathrm{Myr}^{-1} \mathrm{pc}^{-2}$.

By fitting the observed YSOs with a Kroupa IMF, we can extrapolate down to the hydrogen-burning limit to account for the rest of the stellar mass that still remains below the observation detection threshold. Combining this with the estimated pre-main-sequence population from Feigelson et al. (2009) places the current global efficiency of star formation in NGC 6334 at SFE $\sim 0.04_{-0.02}^{+0.03}$ based on a cloud mass of $2.2 \times 10^{5} M_{\odot}$. The conservative estimate for the star formation rate is $8.2_{-4.2}^{+6.3} M_{\odot} \mathrm{Myr}^{-1} \mathrm{pc}^{-2}$ or SFR $\sim 4900_{-2500}^{+3800} M_{\odot} \mathrm{Myr}^{-1}$. Our method assumes the detection of a complete sample of YSOs down to the sensitivity limits of our survey. The true values for the star formation rate may in fact lie closer to, or even above, the upper limits we report based on the uncertainties in the method of converting an incomplete YSO count into a mass determination.

From the estimated mass in Class I YSOs, we find the current rate of star formation in NGC 6334 is $13.0_{-10.4}^{+24.7} M_{\odot} \mathrm{Myr}^{-1}$ $\mathrm{pc}^{-2}$ in the dense star-forming gas within the $A_{V}>8$ contour. The high efficiency and rate of star formation in NGC 6334 indicates that this region may be undergoing a burst of starforming activity, possibly characterized as a "mini-starburst" event.

The methods of data reduction and YSO identification we have derived here will be applied to a sample of five other massive Galactic star-forming complexes observed in a similar fashion with Spitzer IRAC and NEWFIRM. This combined sample will add to the statistical set of Galactic regions with a resolved YSO population characterizing both the high and low ranges of stellar mass. These regions can in turn be directly compared to the Kennicutt-Schmidt relation to assist in bridging the gap between extragalactic star formation and nearby starforming regions.

We acknowledge the assistance of Robert Gutermuth, Vallia Antoniou, Andres Guzman, and Rafael Martinez during the preparation of this manuscript. This work is based on observations made with the Spitzer Space Telescope, which is operated by the Jet Propulsion Laboratory, California Institute 
of Technology, under NASA contract 1407. S.W. acknowledges partial support from NASA grants NNX12AI55G and NNX10AD68G, and JPL-RSA 1369565.

Facilities: Spitzer (IRAC, MIPS)

\section{REFERENCES}

Bally, J. 2008, in Handbook of Star Forming Regions, Volume I, ed. B. Reipurth (San Francisco, CA: ASP), 459

Benjamin, R. A., Churchwell, E., Babler, B. L., et al. 2003, PASP, 115, 953

Brogan, C. L., Hunter, T. R., Cyganowski, C. J., et al. 2009, ApJ, 707, 1

Carey, S., Noriega-Crespo, A., Mizuno, D. R., et al. 2009, PASP, 121, 76

Chavarría, L. A., Allen, L. E., Hora, J. L., Brunt, C. M., \& Fazio, G. G. 2008, ApJ, 682,445

Churchwell, E., Babler, B. L., Meade, M. R., et al. 2009, PASP, 121, 213

Dickinson, M., \& Valdes, F. A. 2009, Guide to NEWFIRM Data Reduction with IRAF, NOAO SDM PL017

Draine, B. T. 2003, ARA\&A, 41, 241

Evans, N. J., II, Dunham, M. M., Jørgensen, J. K., et al. 2009, ApJS, 181, 321

Fazio, G. G., Hora, J. L., Allen, L. E., et al. 2004, ApJS, 154, 10

Feigelson, E. D., Martin, A. L, McNeill, C. J., Broos, P. S., \& Garmire, G. P. 2009, AJ, 132, 227

Fischer, J., Joyce, R. R., Simon, M., \& Simon, T. 1982, ApJ, 258, 165

Gezari, D. Y. 1982, ApJL, 259, L29

Gutermuth, R. A., Megeath, S. T., Muzerolle, J., et al. 2004, ApJS, 154, 374

Gutermuth, R. A., Megeath, S. T., Myers, P. C., et al. 2009, ApJ, 184, 18

Gutermuth, R. A., Pipher, J. L., Megeath, S. T., et al. 2011, ApJ, 739, 84

Harvey, P. M., Huard, T. L., Jørgensen, J. K., et al. 2008, ApJ, 680, 495

Hatchell, J., Terebey, S., Huard, T., et al. 2012, ApJ, 754, 104

Heiderman, A., Evans, N. J., Allen, L. E., Huard, T., \& Heyer, M. 2010, ApJ, 723, 1019

Hughes, S. M. G., \& Wood, P. R. 1990, AJ, 99, 784

Kennicutt, R. C., Jr. 1998, ApJ, 498, 541

Kirk, J. M., Ward-Thompson, D., Di Francesco, J., et al. 2009, ApJS, 185,198

Koenig, X. P., Allen, L. E., Gutermuth, R. A., et al. 2008, ApJ, 688, 1142

Kontizas, E., Dapergolas, A., Morgan, D. H., \& Kontizas, M. 2001, A\&A, 369,932

Kraemer, K. E., \& Jackson, J. M. 1999, ApJS, 124, 439

Krumholz, M. R., \& McKee, C. E. 2008, Natur, 451, 1082

Kryukova, E., Megeath, S. T., Gutermuth, R. A., et al. 2012, AJ, 144, 31

Lada, C. J. 1987, in IAU Symp. 115, Star Forming Regions, ed. M. Peimbert \& J. Jugaku (Dordrecht: Reidel), 1

Lada, C. J., Lombardi, M., \& Alves, J. F. 2009, ApJ, 703, 52

Lada, C. J., Lombardi, M., \& Alves, J. F. 2010, ApJ, 724, 687

Lombardi, M., \& Alves, J. 2001, A\&A, 377, 1023
Makovoz, D., \& Khan, I. 2005, in ASP Conf. Ser. 132, Astronomical Data Analysis Software and Systems XIV, ed. P. L. Shopbell, M. C. Britton, \& R. Ebert (San Francisco: ASP)

Martini, P. 2001, AJ, 121, 598

Matsuura, M., Barlow, M. J., Zijlstra, A. A., et al. 2009, MNRAS, 396, 918

McBreen, B., Fazio, G. G., Stier, M., \& Wright, E. L. 1979, ApJL, 232, L183

McKee, C. F. 1989, ApJ, 345, 782

Megeath, S. T., Allgaier, E., Young, E., et al. 2009, AJ, 137, 4072

Megeath, S. T., Gutermuth, R., Muzerolle, J., et al. 2012, AJ, 144, 192

Meixner, M., Gordon, K. D., Indebetouw, R., et al. 2006, AJ, 132, 2268

Molinari, S., Swinyard, B., Bally, J., et al. 2010, A\&A, 518, L100

Motte, F., Bontemps, S., Hennemann, M., et al. 2012, in SF2A-2012: Proceedings of the Annual meeting of the French Society of Astronomy and Astrophysics, ed. S. Boissier et al., 45

Motte, F., Schilke, P., \& Lis, D. C. 2003, ApJ, 582, 277

Motte, F., Zavagno, A., Bontemps, S., et al. 2010, A\&A, 518, L77

Neckel, T. 1978, A\&A, 69, 51

Persi, P., \& Tapia, M. 2008, Handbook of Star Forming Regions Vol II, ed. B. Reipurth (San Francisco, CA: ASP), 456

Peterson, D. E., Caratti o Garatti, A., Bourke, T. L., et al. 2011, ApJS, 194, 43

Pinheiro, M. C., Copetti, M. V. F., \& Oliveira, V. A. 2010, A\&A, 521, A26

Pompéia, L., Hill, V., Spite, M., et al. 2008, A\&A, 480, 379

Probst, R. G., Gaughan, N., Abraham, M., et al. 2004, Proc. SPIE, 5492, 1716

Rieke, G. H., Young, E. T., Engelbracht, C. W., et al. 2004, ApJS, 154, 25

Robitaille, T. P., Meade, M. R., Babler, B. L., et al. 2008, AJ, 136, 2413

Robitaille, T. P., Whitney, B. A., Indebetouw, R., Wood, K., \& Denzmore, P. 2006, ApJS, 167, 256

Rodriguez, L. F., Canto, J., \& Moran, J. M. 1982, ApJ, 255, 103

Romero, G. A., Schreiber, M. R., Cieza, L. A., et al. 2012, ApJ, 749, 79

Russeil, D., Schneider, N., Anderson, L. D., et al. 2013, A\&A, 554, A42

Russeil, D., Zavagno, A., Adami, C., et al. 2012, A\&A, 538, A142

Schuster, M. T., Marengo, M., \& Patten, B. M. 2006, Proc. SPIE, 6270, 65

Skrutskie, M. F., Cutri, R. M., Stiening, R., et al. 2006, AJ, 131, 1163

Spezzi, L., Vernazza, P., Merín, B., et al. 2011, ApJ, 730, 65

Stetson, P. B. 1987, PASP, 99, 191

Strafella, F., Elia, D., Campeggio, L., et al. 2010, ApJ, 719, 9

Straw, S. M., \& Hyland, A. R. 1989, ApJ, 340, 318

Swaters, R. A., Valdes, F. \& Dickinson, M. E. 2009, in ASP Conf. Ser 411, Astronomical Data Analysis Software and Systems XVIII, ed. D. A. Bohlender, D. Durand, \& P. Wodlwer (San Francisco, CA: ASP), 506

Tapia, M., Persi, P., \& Roth, M. 1996, A\&A, 316, 102

van Aarle, E., van Winckel, H., Lloyd Evans, T., et al. 2011, A\&A, 530, A90 van Winckel, H. 2003, ARA\&A, 41, 391

Werner, M. W., Roellig, T. L., Low, F. J., et al. 2004, ApJS, 154, 1

Woods, P. M., Oliveira, J. M., Kemper, F., et al. 2011, MNRAS, 411, 1597

Zernickel, A., Schilke, P., \& Smith, R. J. 2013, A\&A, 554, L2 